Elskamp, F.; Kruggel-Emden, $\mathrm{H}$.

\title{
Extension of process models to predict batch screening results under the influence of moisture based on DEM simulations
}

Journal article | Accepted manuscript (Postprint)

This version is available at https://doi.org/10.14279/depositonce-8318



Elskamp, F., \& Kruggel-Emden, H. (2019). Extension of process models to predict batch screening results under the influence of moisture based on DEM simulations. Powder Technology, 342, 698-713.

https://doi.org/10.1016/j.powtec.2018.10.039 


\section{Extension of process models to predict batch screening}

2 results under the influence of moisture based on DEM

\section{3 simulations}

4 Frederik Elskamp ${ }^{1 \star}$, Harald Kruggel-Emden ${ }^{1}$

$5{ }^{1}$ Mechanical Process Engineering and Solids Processing, Technische Universität Berlin, Ernst-

6 Reuter-Platz 1, D-10587 Berlin, Germany

7 *Corresponding author. Tel.: +49-30-314-23496; Fax: +49-30-314-26432

8 E-mail address: frederik.elskamp@tu-berlin.de

\section{Abstract}

Screening is a technical simple but still not fully understood process step, which can be used in a wide field of applications to separate bulk materials according to their particle sizes. A severe issue in screening technologies is that particles frequently prevail in moist conditions, due to effects related to the environment, the material or the process. This is often not preventable, although it is not preferred due to attractive forces altering the screening efficiency. For the design of dry screening processes, phenomenological models and detailed particle-based simulation approaches like the discrete element method (DEM) are available. The latter method has recently been extended and validated against experiments to calculate forces caused by liquid bridges formed out between particles or walls close to each other to meet the requirements to tackle real particle systems under moist conditions. In the investigation here, batch screening under the influence of moisture involving different sized glass spheres is investigated numerically with DEM simulations and by using process models. Therein, the related subprocesses stratification and passage as well as the influence of the operating parameters and the liquid amount on the fraction retained per size class are examined. Existing phenomenological process models, which can be applied efficiently for industrial applications due to their short calculation time, are extended to represent batch screening processes under moist conditions for the first time. Therefore, a benchmark is realized in which the fraction retained per size class over time for discontinuous screening under the influence of various amounts of liquid and different mechanical agitations obtained by DEM simulations and process models is compared. In this context, the process models are first adjusted to fit related simulation results and later used in a novel method to predict the outcome of screening with different operating parameters and liquid amounts. Thereby, process models, which consider the subprocesses stratification and passage, predict screening results for process parameters requiring interpolation or extrapolation in the 
investigated range very well. As a consequence, newly derived process models can function as prototypes to be applied in dynamic process simulation frameworks.

Keywords: Discrete element method; Process model; Liquid bridges; Screening; Moisture

\section{Introduction}

Screening is a simple but major process step used in a wide field of industrial applications to perform a classification of bulk material into particles of requested size classes [1,2]. Most of performed investigations on screening only considered dry particles, whereas screening under the influence of liquid has rarely been studied until now. The only exception are a few pure experimental investigations considering different amounts of liquid [3-5] under process specific conditions for particular applications.

To study screening and its subprocesses under moist or wet conditions in detail without performing extensive experimental tests, the discrete element method (DEM) can be used. This method was first introduced by Cundall and Strack [6] and proved as a suitable tool in various investigations on screening [7-9]. In these studies, the fluid was omitted or the material was assumed as dry resulting in a mostly undisturbed transport, stratification and passage of the particles. In contrast, some researchers concentrated on wet screening applications and coupled the DEM with methods to model the fluid flow like cell-based computational fluid dynamics [10] or particle-based smoothed particle hydrodynamics [11]. In wet screening processes, the liquid can support the transport of fine particles through the apertures. For screening, dry or completely wet conditions are preferred over conditions where material is only influenced by a slight amount of water, where the particles can adhere to each other and the screening efficiency is reduced [4,12]. For more details on this, Zhu et al. [13] provide an overview of theoretical developments of discrete particle simulations of dry and wet particulate systems.

A small amount of water in the screening process conveyed from preceding process steps cannot always be prevented and consequently, the impact on the screening process has to be better understood and the consequences for following process steps should be made ascertainable. Therefore, a relevant state-of-the-art task is the development of an efficient and robust dynamic process simulation framework [14], where a dynamic screening model is an essential process step. In this framework, the results of a screening model can be influenced by liquid or material under moist conditions from other process steps. For this reason, the extension of an appropriate phenomenological process model for screening under the influence of moisture is inevitable. Besides the possibility to consider a small amount of water, this model should account for different particle sizes, various operating conditions and it should 
be able to represent batch screening with its inherent transient nature and a possibly thick particle bed with multiple layers at the beginning of the screening process. The DEM, extended by forces, which arise from the presence of liquid, seems to be suitable to study screening under the influence of liquid as well as to provide data to adjust and to test respective process models.

Liquid bridges including their formation, the resulting forces and the rupture event were studied experimentally over decades by several researchers [15-19]. In other investigations in the recent past, the impact behavior of wet particles on dry surfaces or vice versa was studied to obtain the restitution coefficient under these conditions and to apply it in DEM simulations [2022]. However, in many studies [23-27] and in the investigation here, the applied DEM is extended with respective force models to simulate screening under moist conditions.

The primarily studied forces arising from a liquid bridge contact are the capillary and the viscous forces. While capillary forces are dominant in systems with slow particle movements and low liquid viscosities, the importance of the viscous forces increases for fast moving particles involving liquids with high viscosities. The capillary forces can either be obtained by the energetic method based on the total interfacial energy or by summing up the pressure and tension terms from the meniscus profile based on the Young-Laplace equation $[16,28]$. Latter can be subdivided in the neck [29] and boundary method [30]. One example where the capillary force is calculated between two spherical bodies with the boundary method is the liquid bridge model by Weigert and Ripperger [18], where the bridge volume is related to the half-filling angle. In contrast, the frequently used models by Willett et al. [17] and Rabinovich et al. [15] both applicable for unequal sized spheres are representatives of the neck method. Rabinovich et al. [15] also introduced equations for different wettability of contact partners and for contacts between spherical particles and walls. Gladkyy and Schwarze [31] benchmarked these capillary bridge models by applying them in a DEM framework, whereby the latter two models (Willett et al. [17] and Rabinovich et al. [15]) reveal results close to the presented experimental data. In order to provide tractable calculations for the capillary forces, Lian and Seville [32] developed closed-form equations, which can be applied for capillary bridges containing varying amounts of liquid formed out between unequal sized spheres with different contact angles.

For particle systems with a high liquid viscosity or large interparticle velocities, the consideration of the viscous forces is necessary. A commonly used liquid bridge viscosity model in the normal direction was proposed by Adams and Perchard [33] and later extended and applied in a DEM framework by Pitois et al. [34], who studied the viscosity effects of a liquid bridge between two moving spheres. Furthermore, Goldmann et al. [35] introduced an often applied tangential viscosity model for liquid bridge contacts. 
In addition to the acting forces, several researchers [36-38] studied the formation process of

104 a liquid bridge including its shape and the containing liquid volume as well as the liquid redistribution after rupture of a bridge. The rupture conditions were intensively addressed by Willett et al. [17] and several other researchers like Pitois et al. [39] who further developed the up to then static to a dynamic rupture model.

108 Several studies of larger particulate systems were performed for 2D [40] and 3D [41] like 109 packed beds or rotating drums with uniform spheres [24-26] as well as mixing processes with 110 unequal sized spheres [23,38,42]. Besides some exceptions [10], such investigations are 111 mostly lacking for screening processes. In a previous investigation by the authors [27], an 112 extension of the DEM including a validation for screening under the influence of moisture has 113 been performed. Therein, only a small amount of liquid is applied to ensure individual capillary 114 bridges without liquid in the pores inbetween the particles.

115 The derivation and verification of simpler and less computing-intensive phenomenological 116 screening process models is also possible with the DEM. One of the first, who applied the DEM 117 instead of performing extensive experiments for this purpose were Shimosaka et al. [43]. A 118 comprehensive benchmark of phenomenological screening process models based on the 119 results of batch screening processes modelled by the DEM was performed by Elskamp et al. 120 [44]. These models were not applicable for screening under moist conditions and limited in 121 predictability. In a very recent investigation, Dong et al. [45] introduced a model based on the 122 work by Subasinghe et al. [46], which provides the ability to predict the outcome for continuous 123 screening processes valid for a thin particle bed and square [45] as well as rectangular 124 apertures [47]. In contrast to continuous screening, in batch screening investigation, the 125 assumption of a thin particle bed is invalid. This results in the necessity of considering 126 stratification arising from thicker particle beds in addition to the passage process according to 127 the works by Subasinghe et al. [48] and Soldinger [49,50] in future investigations.

128 In the present study, first, batch screening under the influence of moisture and different 129 operating conditions involving spherical particles is investigated numerically. Based on these 130 DEM simulation results, a comparative study of selected phenomenological screening process 131 models (compare $[44,51]$ ) is conducted. As novelty, these models are extended for the 132 representation and prediction of discontinuous screening under various amounts of liquid and 133 different mechanical agitations. As a result, the extended batch screening models will be 134 applied in dynamic flowsheet simulations of solids processes (Dyssol) together with other 135 process models to simulate and predict the outcome of connected processes in further 136 investigations [52]. 


\section{Numerical method}

138 In this section, the discrete element method including the applied contact and liquid bridge

139 force laws as well as the liquid distribution are briefly described.

\subsection{The discrete element method}

141 The tracking of particles and the calculation of their translational and rotational motion in 142 various systems can be realized by utilizing the DEM $[13,53]$. Therefore, the Newton's and 143 Euler's equations are integrated

$$
\begin{aligned}
& m_{i} \frac{d^{2} \vec{x}_{i}}{d t^{2}}=\vec{F}_{i}^{c}+\vec{F}_{i}^{l}+m_{i} \vec{g}, \\
& I_{i} \frac{d \vec{\omega}_{i}}{d t}=\vec{M}_{i}
\end{aligned}
$$

144 with particle mass $m_{i}$, particle acceleration $d^{2} \vec{x}_{i} / d t^{2}$, contact force $\vec{F}_{i}^{c}$, liquid bridge force $\vec{F}_{i}^{l}$, 145 gravitational force $m_{i} \vec{g}$, moment of inertia $I_{i}$, angular acceleration $d \vec{\omega}_{i} / d t$, angular velocity $\vec{\omega}_{i}$ 146 and external moments resulting out of contact and liquid bridge forces $\vec{M}_{i}$. Both equations can 147 be solved by using explicit integration schemes (compare e.g. [54]). Fig. 1a shows a sketch of 148 two colliding different sized spheres $i$ and $j$.

a

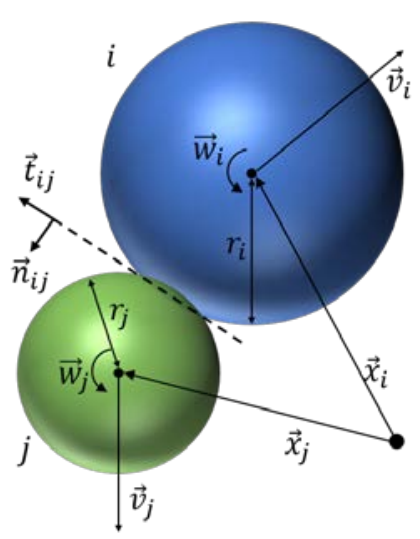

b





Fig. 1: (a) A collision of two spherical particles and liquid bridges having formed out between (b) spherical particles of different sizes, (c) a particle with a large wall with $r_{w}>r_{i}$ (side wall) as well as (d) with a small wall of $r_{w}<r_{i}$ (screen wire).

151 For such a contact, the resulting contact force consists of normal and tangential forces

$$
\vec{F}_{i j}^{c}=\vec{F}_{i j}^{c n}+\vec{F}_{i j}^{c t},
$$

where a linear spring damper model is used to obtain the normal component as 


$$
\vec{F}_{i j}^{c n}=k^{n} \delta_{i j} \vec{n}_{i j}+\gamma^{n} \vec{v}_{i j}^{n}
$$

153

154

$$
\vec{F}_{i j}^{l}=\vec{F}_{i j}^{c a p}+\vec{F}_{i j}^{n v i s}+\vec{F}_{i j}^{t v i s}
$$

where $k^{n}$ is the spring stiffness, $\delta_{i j}$ the virtual overlap, $\vec{n}_{i j}$ a normal vector, $\gamma^{n}$ a damping coefficient and $\vec{v}_{i j}^{n}$ the normal velocity at the contact point [55]. The normal coefficient of restitution between particles $e_{P P}^{n}$ as well as particles and walls $e_{P W}^{n}$ under dry conditions is determined by $k^{n}$ and $\gamma^{n}$. A linear spring limited by the Coulomb condition is applied to obtain the tangential forces, leading to

$$
\vec{F}_{i j}^{c t}=-\min \left(k^{t}\left|\vec{\xi}_{i j}\right|, \mu_{C}\left|\vec{F}_{i j}^{n}\right|\right) \vec{t}_{i j}
$$

where $k^{t}$ is the tangential stiffness of a linear spring, $\mu_{C}$ is the friction coefficient, $\vec{\xi}_{i j}$ is the relative tangential displacement and $\vec{t}_{i j}$ is the tangential unit vector [56].

\subsection{Liquid bridges in the discrete element method}

In this investigation, only a small and uniformly distributed amount of liquid is added to the particles to ensure the pendular state with individual liquid bridges between pairs of particles. Several adhering forces result out of the existence of these liquid bridges, of which the capillary and the viscous forces are applied in this work. The total liquid bridge force is obtained as

where $\vec{F}_{i j}^{c a p}$ is the capillary force and $\vec{F}_{i j}^{\text {nvis }}$ as well as $\vec{F}_{i j}^{t v i s}$ are the viscous forces in normal 166 and tangential direction, respectively. An extension is made for the external moment $\vec{M}_{i}$ 167 (compare eq. (2)), which is now the sum of the moments due to a contact $\vec{M}_{C, i}$ and a liquid 168 bridge $\vec{M}_{L, i}=\vec{r} \times \vec{F}_{i}^{\text {tvis }}$.

169 A liquid bridge forms out between two particles $i$ and $j$ or a particle and a wall when they get 170 into contact under the influence of moisture (Figs. 1b-d). For two spherical particles (compare 171 Fig. 1b), the liquid volumes contributing from each particle are combined to the total volume of 172 the liquid bridge $V_{l b}$ as

$$
V_{l b}=V_{i}+V_{j}=\frac{L_{i}}{2}\left(1-\sqrt{1-\frac{r_{j}^{2}}{\left(r_{i}+r_{j}\right)^{2}}}\right)+\frac{L_{j}}{2}\left(1-\sqrt{1-\frac{r_{i}^{2}}{\left(r_{i}+r_{j}\right)^{2}}}\right)
$$

173 where $L_{i}$ and $L_{j}$ are the total liquid volumes present on particles $i$ and $j$ [37]. 
174 The volume of a liquid bridge between a particle $i$ and a wall is $V_{l b}=V_{i}+V_{w}$. In case of a wall, 175 which is extending larger than the diameter of the particles (compare Fig. 1c), the liquid volume

$176 V_{i}$ contributed from the particle is assumed to be

$$
V_{i}=\frac{L_{i}}{2}(1-\sqrt{0.75})
$$

177 The liquid contributed from the wall is calculated as

$$
V_{w}=W_{l f} \frac{\pi}{4} r_{i}^{2}
$$

178 where $W_{l f}$ is the liquid film thickness on the wall.

179 For the case of a liquid bridge contact between a particle and a screen wire (compare Fig. 1d) 180 the liquid that contributes from the particle is calculated as

$$
V_{i}=\frac{L_{i}}{6 r_{i}} r_{w}
$$

181 The liquid contributed from the screen wire is assumed as

$$
V_{w}=W_{l f} r_{i} 2 r_{w}
$$

182

where $r_{i} 2 r_{w}$ is the projection surface of the particle's spherical cap on the wall. Note that the liquid bridge volume is assumed as constant as long as it exists. A detailed explanation and derivation of the equations concerning the formation of a liquid bridge can be found in a previous work by the authors [27].

In the investigation here, the applied capillary force is based on the models used by Rabinovich et al. [15] and Pitois et al. [34], where the force is calculated at the neck of the liquid bridge 188 (compare Figs. 1b-d). Therein, the capillary forces for a liquid bridge between two particles $i$ 189 and $j$ as well as between a particle and a wall are determined as

$$
\begin{aligned}
& \vec{F}_{i j p p}^{c a p}=\left(-\frac{\pi \sigma r_{e f f}\left(\cos \theta_{i}+\cos \theta_{j}\right)}{1+1 /\left(\sqrt{1+\frac{2 V_{l i q}}{\left(\pi r_{e f f} S^{2}\right)}}-1\right)}-2 \pi \sigma r_{e f f} \sin \left(\theta_{i j}\right) \sin \left(\theta_{i j}+\varphi\right)\right) \vec{n}_{i j}, \\
& \vec{F}_{i p w}^{\text {cap }}=\left(-\frac{2 \pi \sigma r_{i}\left(\cos \theta_{i}+\cos \theta_{w}\right)}{1+S \sqrt{\pi r_{i} / V_{l b}}}-2 \pi \sigma r_{i} \sin \left(\theta_{i w}\right) \sin \left(\theta_{i w}+\varphi\right)\right) \vec{n}_{i w},
\end{aligned}
$$

190 where $\sigma$ is the surface tension coefficient, $r_{e f f}=2 r_{i} r_{j} /\left(r_{i}+r_{j}\right)$ is the effective radius, $\theta_{i}, \theta_{j}$ 191 and $\theta_{w}$ are the static contact angles of the particles $i, j$ and a wall, respectively. The parameters 
$\theta_{i j}=\left(\theta_{i}+\theta_{j}\right) / 2$ and $\theta_{i w}=\left(\theta_{i}+\theta_{w}\right) / 2$ are the mean contact angles of two particles as well

193 as of a particle and a wall, respectively (compare [57]). $S$ is the separation distance between 194 two contact partners and $\varphi$ is the half filling angle, obtained in the case of two spherical 195 particles as

$$
\varphi=\sqrt{S / r_{e f f}\left(-1+\sqrt{1+2 V_{l b} /\left(\pi r_{e f f} S^{2}\right)}\right)} .
$$

196 For a particle and a wall, $\varphi$ is given as

$$
\varphi=\sqrt{2 S / r_{i} \sqrt{1+V_{l b} /\left(\pi r_{i} S^{2}\right)}}
$$

$$
\vec{F}_{i j}^{n v i s}=-\frac{6 \pi \eta r_{r e f f}^{2} \vec{v}_{r}^{n}}{S}\left(1-1 / \sqrt{\left(1+V_{l b} /\left(\pi r_{r e f f} S^{2}\right)\right)}\right)^{2},
$$

201 where $\eta$ is the liquid dynamic viscosity, $r_{\text {reff }}=r_{i} r_{j} /\left(r_{i}+r_{j}\right)$ is the reduced effective radius and $202 \vec{v}_{r}^{n}=\left(\left(\vec{v}_{i}-\vec{v}_{j}\right) \cdot \vec{n}_{i j}\right) \vec{n}_{i j}$ is the relative normal velocity of particles with the velocities $\vec{v}_{i}$ and $\vec{v}_{j}$. 203 For the tangential viscous forces, Goldman et al. [35] proposed the following equations valid 204

$$
\begin{aligned}
& \vec{F}_{i j}^{t v i s}=-6 \pi \eta r_{\text {reff }}\left(\frac{8}{15} \ln \frac{r_{\text {reff }}}{S}+0.9588\right) \vec{v}_{r}^{t}-6 \pi \eta r_{r e f f}\left(\frac{2}{15} \ln \frac{r_{r e f f}}{S}-0.2526\right) \vec{\omega}_{r} \times \vec{n}_{i j}, \\
& \vec{F}_{i j}^{t v i s}=-6 \pi \eta r_{\text {reff }}\left(\frac{8}{15} \ln \frac{r_{r e f f}}{S}+0.9588\right) \vec{v}_{r}^{t} \\
& -\frac{6 \pi \eta r_{\text {reff }}}{8}\left(\frac{r_{\text {reff }}}{S+r_{\text {reff }}}\right)^{4}\left(1-\frac{3 r_{\text {reff }}}{8\left(S+r_{\text {reff }}\right)}\right) \vec{\omega}_{r} \times \vec{n}_{i j},
\end{aligned}
$$
with $\vec{v}_{r}^{t}=\vec{v}_{i}-\vec{v}_{j}-\vec{v}_{r}^{n}$ as the tangential relative velocity from the translational motion and $\vec{\omega}_{r}=$ $r_{i} \vec{\omega}_{i}+r_{j} \vec{\omega}_{j}$ as relative rotational velocity of the spheres. Note that a minimum separation distance $S_{\min }=0.001 r_{\text {reff }}$ is added to $S$ to prevent that the viscous forces tend to infinity when $S$ approaches zero (compare e.g. [58]). 
When the distance $S$ between two contact partners reaches a respective length, the liquid bridge ruptures. Based on the work by Willett et al. [17] the rupture distance is calculated as

$$
S_{\text {rup }}=r_{\text {eff }}\left(1+\left(0.125\left(\theta_{i}+\theta_{j}\right)\right)\left(1+\frac{r_{j}}{r_{i}}\right)\right)\left(\left(\frac{V_{l b}}{r_{e f f}^{3}}\right)^{1 / 3}+\left(\frac{r_{j}}{2 r_{i}}-\frac{2}{5}\right)\left(\frac{V_{l b}}{r_{e f f}^{3}}\right)^{2 / 3}\right),
$$

211 with $r_{i} \geq r_{j}$. Additionally, in this work, the dynamic rupture distance

$$
S_{\text {rup }, \text { dyn }}=S_{\text {rup }}\left(1+\sqrt{\left(\frac{\left(\vec{v}_{i}-\vec{v}_{j}\right) \eta}{\sigma}\right)}\right)
$$

212 proposed by Pitois et al. [39] is applied to account for the rupture distance dependency of the 213 particle velocity.

214 Note that the rupture of a liquid bridge occurs at its thinnest point. This point is somewhere 215 located between the contact partners depending on the particle size, the contact angles and 216 the liquid bridge volume. This rupture location is decisive for the liquid share, which is received by each contact partner after a rupture event. More details on the liquid distribution, the transfer ratio and the contact angles as well as a numerical validation of the liquid bridge forces with data from literature can be found in a previous publication by the authors [27].

\section{Extended phenomenological screening process models}

Phenomenological screening process models can be used for the time resolved representation of the particle size separation during a batch screening process. This can be achieved by probabilistic theoretical $[45,46,59]$ and kinetic $[48,60,61]$ models which are either directly applicable to discontinuous screening or they can be used for batch screening by replacing length / by time $t$ in the equations of the respective models (compare [44]).

Kinetic models are based on first order kinetics, whereas probabilistic models need additional parameters like the probability of particles to pass an aperture e.g. by Gaudin [62]. Several probabilistic models consider the screen motion, the aperture shape and size as well as the particle composition and shape [45-47,59]. In addition, some more complex phenomenological screening models take the opposing subprocesses stratification and particle passage into account by providing additional parameters [48-50]. However, besides some exceptions

$232[45,47]$, most of the existing models lack the ability to predict the outcome of screening processes with different mechanical agitations or bulk characteristics satisfactorily.

Table 1 provides an overview of all applied screening process models in this investigation, which are extended for screening under the influence of moisture and for the prediction of 
screening results with different operating parameters and liquid amounts as novelty of this work. They are titled by the author's names and a model number and include the major equations as well as the used model parameters. A more detailed description of all investigated models for dry screening can be found in Elskamp and Kruggel-Emden [44] or in the respective publications. All investigated models should represent the fraction retained per particle size class $i$ over time which is

$$
Y_{i}=Y_{i}(t)=m_{p, l, i} / m_{p, l, i, 0}
$$

242 where $m_{p, l, i, 0}$ is the initial fractional mass of the particles at $t=0 \mathrm{~s}$ and $m_{p, l, i}$ is the remaining

243 fractional mass of the particles at time $t$. Note that both masses include the particles plus the 244 liquid assigned to the particles.

245 Table 1: Major equations of the extended and applied phenomenological screening process models.

\begin{tabular}{|c|c|c|}
\hline $\begin{array}{l}\text { Model number } \\
\text { and origin }\end{array}$ & Major equations & $\begin{array}{l}\text { Adjustable } \\
\text { parameters }\end{array}$ \\
\hline $\begin{array}{l}\text { 1. Dong et al. [45] } \\
\text { (based on } \\
\text { Subasinghe et al. } \\
\text { [46]) }\end{array}$ & $\begin{array}{c}Y_{i}=\left(1-P_{i}\right)^{N_{i}} \\
P_{i}=\left(a-d_{i}\right)^{2} /(a+w)^{2} \\
a \text { : aperture size } W: \text { wire diameter; } d_{i}: \text { particle diameter } \\
N_{i}=k\left(\frac{A f(1-M)^{\gamma}}{\sqrt{d_{i} g}}\right)^{\alpha} \frac{t}{t_{\text {end }}}\end{array}$ & $k, \alpha, \gamma$ \\
\hline $\begin{array}{l}\text { 2. Subasinghe et al. } \\
\text { [48] }\end{array}$ & $\begin{array}{c}Y_{i}=\left(k_{s, i} \exp \left(-k_{p, i} t\right)-k_{p, i} \exp \left(-k_{s, i} t\right)\right) /\left(k_{s, i}-k_{p, i}\right) \\
k_{s, i}=k_{s}\left(\frac{A f(1-M)^{\gamma}}{\sqrt{d_{i} g} \frac{d_{i}}{d_{a v}}}\right)^{\alpha} \\
k_{p, i}=k_{p}\left(\frac{A f(1-M)^{\delta}}{\sqrt{d_{i} g} \frac{d_{i}}{a}}\right)^{\beta}\end{array}$ & $k_{s}, k_{p}, \alpha, \beta, \gamma, \delta$ \\
\hline 3. Soldinger [50] &  & $k_{s}, k_{p}, \alpha, \beta, \gamma, \delta$ \\
\hline
\end{tabular}

247 Subasinghe et al. [46] proposed a probabilistic screening model, where the probability $P_{i}$ for a 248 particle to remain on the screen after $N_{i}$ attempts is

$$
P_{i}\left(N_{i}\right)=\left(1-p_{i}\right)^{N_{i}}
$$


249 where $p_{i}$ is the probability of the particle to pass the apertures in a single attempt, which is 250 calculated as

$$
p_{i}=\frac{\left(a-d_{i}\right)\left(a \cos (\tau)-d_{i}\right)}{(a+w)^{2} \cos (\tau)}
$$

251 where $a$ is the aperture size, $d_{i}$ the particle diameter, $w$ the wire diameter and $\tau$ the inclination 252 of the screen. For a horizontal batch screen, this correlation is simplified to

$$
p_{i}=\frac{\left(a-d_{i}\right)^{2}}{(a+w)^{2}}
$$

253 For a bulk of particles, the fraction retained per size class is calculated similar to eq. (22), by 254 using the average probability $p_{i}$ per size class. The probability is multiplied with the ratio of the 255 mass of one particle to the total initial mass fraction leading to

$$
Y_{i}=\left(1-p_{i} \frac{m_{p, l, i}}{m_{p, l, i, 0}}\right)^{N_{i}} .
$$

256 The amplitude $A$ and the frequency $f$ influence the motion of the particles on the screen. The 257 motion is also dependent on the particle diameter $d_{i}$. For a continuously operated screen with 258 a thin particle bed, Dong et al. [45] found out that $N_{i}$ is lower for larger $A \cdot f$ as well as for a larger $259 \tau$ and $N_{i}$ decreases nearly linearly for an increasing particle size $d_{i}$. Based on these 260 considerations and dimensional analysis, the number of attempts $N_{i}$ is represented according 261 to Dong et al. [45] by

$$
N_{i}=k\left(\frac{A f}{\sqrt{d_{i} g}}\right)^{\alpha}\left(\frac{d_{i}}{L}\right)^{\beta} \tau^{\delta},
$$

262 with the fitting parameters $k, \alpha, \beta, \delta$, the gravitational force $g$ and the total length of the deck $L$. 263 In the investigation here, the last part of the equation has to be removed for a horizontal screen. 264 In case of batch screening, the length $L$ has to be replaced by the time $t$. To maintain a 265 dimensionless value, the actual point in time $t$ is set in relation to the total simulation time $t_{\text {end }}$ 266 resulting in

$$
N_{i}=k\left(\frac{A f}{\sqrt{d_{i} g}}\right)^{\alpha} \frac{t}{t_{\text {end }}} .
$$

In case of screening under the influence of moisture, the liquid amount $M$ influences the motion of the particles on the screen by reducing their motion. Note that the term 1 - $M$ is used to apply 
269 this equation under moist and dry conditions $(M=0 \%)$. Since the influence of the liquid 270 amount is not in advance quantifiable, a third fitting parameter $\gamma$ has to be added, leading to

$$
N_{i}=k\left(\frac{A f(1-M)^{\gamma}}{\sqrt{d_{i} g}}\right)^{\alpha} \frac{t}{t_{\text {end }}}
$$

271 which is used in combination with eq. (24) and eq. (25) and referred to as model No. 1 in the 272 following.

273 The basis for kinetic screening models is the "first-order rate law" in which the explicit equation 274 for the fraction retained per size class of particles remaining above the screen is

$$
Y_{i}(t)=\exp \left(-k_{i} t\right)
$$

275 where the screening rate constant $k_{i}$ is an adjustable parameter.

276 Subasinghe et al. [48] also introduced a kinetic model, where besides the passage of the undersized particles, the stratification of the small particles through the coarse material is considered. A detailed derivation can be found in their work, leading to the following equation 279 for the fraction retained per particle size class $i$

$$
Y_{i}(L)=\left(k_{s, i} \exp \left(-k_{p, i} L\right)-k_{p, i} \exp \left(-k_{s, i} L\right)\right) /\left(k_{s, i}-k_{p, i}\right)
$$

280 Instead of the screening rate constant $k_{i}$, the adjustable parameters $k_{s, i}$ and $k_{p, i}$ were 281 introduced. The parameter $k_{s, i}$ is used to describe the fraction retained of particles above the 282 screen and not in contact with it, whereas $k_{p, i}$ is applied to consider the fraction retained of 283 particles above the screen that are in contact with the screen. To apply eq. (30) for the 284 representation of batch screening processes, the screen length $L$ is exchanged by the time $t$, 285 leading to

$$
Y_{i}(t)=\left(k_{s, i} \exp \left(-k_{p, i} t\right)-k_{p, i} \exp \left(-k_{s, i} t\right)\right) /\left(k_{s, i}-k_{p, i}\right)
$$

286 In order to make the model and its adjustable parameters $k_{s, i}$ and $k_{p, i}$ dependent on the screen 287 motion and the particle sizes according to model No. 1, the following equations are introduced

$$
k_{s, i}=k_{s}\left(\frac{A f}{\sqrt{d_{i} g} \frac{d_{i}}{d_{a v}}}\right)^{\alpha}
$$




$$
k_{p, i}=k_{p}\left(\frac{A f}{\sqrt{d_{i} g} \frac{d_{i}}{a}}\right)^{\beta}
$$

288 where $d_{a v}=\frac{1}{n} \sum_{i=1}^{n} d_{i}$ is the average particle size. The relations $\frac{d_{i}}{d_{a v}}$ and $\frac{d_{i}}{a}$ are additionally 289 applied to consider the particle composition and to compensate for the lack of the particle 290 passage probability.

291 For screening under the influence of moisture, the motion of the particles on the screen is 292 influenced, which is realized similarly like in model No. 1, leading to

$$
\begin{aligned}
& k_{s, i}=k_{s}\left(\frac{A f(1-M)^{\gamma}}{\sqrt{d_{i} g} \frac{d_{i}}{d_{a v}}}\right)^{\alpha}, \\
& k_{p, i}=k_{p}\left(\frac{A f(1-M)^{\delta}}{\sqrt{d_{i} g} \frac{d_{i}}{a}}\right)^{\beta} .
\end{aligned}
$$

293 This model is referred to as model No. 2 in the following.

294 In the investigations by Soldinger [49,50], a bottom layer of fine material is introduced besides 295 the subprocesses stratification and passage. This bottom layer consists of all undersized 296 particles directly on the screen surface and those that have the possibility to reach the screen 297 without being blocked by other particles in their way. In contrast to her first investigation [49], 298 Soldinger subdivided the undersized particles in different size classes $i$ in her following studies 299 (compare e.g. [50]). The fractional mass of undersized particles per size class in the bottom 300 layer $B_{i}$ changes over time due to the concurrent mass streams $\dot{S}_{i}$ and $\dot{R}_{i}$, which are the 301 particles stratifying to the bottom layer and those passing the apertures and thus leaving the 302 bottom layer, respectively. This procedure is described by

$$
\dot{B}_{\imath}=\dot{S}_{i}-\dot{R}_{i}=\frac{B_{i, j+1}-B_{i, j}}{t_{j+1}-t_{j}}=\frac{S_{i, j+1}-S_{i, j}}{t_{j+1}-t_{j}}-\frac{Y_{i, j}-Y_{i, j+1}}{t_{j+1}-t_{j}}
$$

303 where $\Delta t=t_{j+1}-t_{j}$ is a fixed, discrete time step.

304 The fractional mass stream of stratified particles of class $i$ can be calculated as

$$
\dot{S}_{i}=\left(S_{i, j+1}-S_{i, j}\right) /\left(t_{j+1}-t_{j}\right)=\mathrm{c}_{i, j}\left(S_{i, \infty}-S_{i, j}\right),
$$




$$
B_{i, j+1}=B_{i, j}+\left(\mathrm{c}_{i, j}\left(S_{i, \infty}-S_{i, j}\right)-k_{i, j} B_{i, j}\right)\left(t_{j+1}-t_{j}\right), \quad \dot{B}=\sum_{i=1}^{n} \dot{B}_{i},
$$

308 and

$$
\dot{R}_{i}=\left(Y_{i, j}-Y_{i, j+1}\right) /\left(t_{j+1}-t_{j}\right)=\mathrm{k}_{i, j} B_{i, j}, \quad \dot{R}=\sum_{i=1}^{n} \dot{R}_{i},
$$

respectively. $B_{i, j}$ is limited by $B_{p}=B_{m P} / m_{0}$, where $B_{m P}$ is the mass of the bottom layer. Therein, $k_{i, j} B_{i, j}$ is exchanged by $B_{p} \cdot k_{i, j} \cdot B_{i, j} / B_{j}$ when $B_{j}>B_{p}$. In this investigation, the

passage parameter $k_{i, j}=b_{i}\left(S_{i, \infty}-\left(1-Y_{i, j}\right)\right)$ is influenced by the parameter $b_{i}$ similar to the first model by Soldinger [49]. To make the passage parameter additionally dependent on the screen motion and the liquid amount, $b_{i}$ is determined according to model No. 2 as

$$
b_{i}=k_{p}\left(\frac{A f(1-M)^{\delta}}{\sqrt{d_{i} g} \frac{d_{i}}{a}}\right)^{\beta}
$$

314 in this investigation. The rate of stratification is obtained by the time dependent parameter

$$
c_{i, j}=\left(c_{q, i, j} \cdot c_{d, i, j}\right) /\left(H_{t, j} / d_{a v, c}\right)
$$

where the average diameter of the coarse particles $d_{a v, c}$ as well as the thickness of the top layers in the particle bed $H_{t, j}=M_{t, j} /\left(W^{2} \cdot \rho_{b}\right)$, with the mass of material in the top layer $M_{t, j}$, the length and width of a quadratic screen $W$ and the bulk density $\rho_{b}$ assumed as constant are taken into account. In addition, $c_{q, i, j}$ and $c_{d, i, j}$ are both dependent on the proportion of fine material in the top layer which is obtained by

$$
q_{i, j}=\left(m_{0} \cdot \sum_{i=1}^{n}\left(S_{i, \infty}-S_{i, j}\right)\right) /\left(M_{0}\left(1-Q_{0}\right)+m_{0} \cdot \sum_{i=1}^{n}\left(S_{i, \infty}-S_{i, j}\right)\right) .
$$

320 The parameter $c_{d, i, j}=\exp \left(-2.5 \cdot V_{i, p a r t} / V_{a v, t, j}\right)$ is obtained with $V_{i, p a r t}$ and $V_{a v, t, j}$ which are 321 the volume of the respective particle and the average volume of particles in the top layer, 322 respectively. The parameter $c_{q, i, j}$ is obtained by $c_{q, i, j}=w_{q, i} \exp \left(-\left(2 q_{i, j}\right)^{5}\right)$. Soldinger [50] 323 expected that $w_{q, i}$ increases for an increase in screen motion due to a larger frequency or 324 amplitude. In addition, it is assumed that a larger amount of liquid reduces $w_{q, i}$. Therefore, in 325 the investigation here, this parameter is determined according to model No. 2 as 
$w_{q, i}=k_{s}\left(\frac{A f(1-M)^{\gamma}}{\sqrt{d_{i} g} \frac{d_{i}}{d_{a v}}}\right)^{\alpha}$.

This model is referred to as model No. 3 in the following.

\section{Numerical setup and simulation parameters}

For the DEM simulations in this study, a batch screening apparatus is modeled according to a modified "Haver and Boecker EML digital plus" batch screen tower with a circular screen surface with a woven mesh and square apertures, which is applicable for dry and wet screening 331 (compare Fig. 2).



Fig. 2: Batch screening apparatus and close-up of the applied screen surface in the DEM simulations. Particles are coloured according to (left) size at $t=0 \mathrm{~s}$ and (right) liquid amount at $t=3 \mathrm{~s}$.

334 A feed bin is placed over the screen apparatus without direct contact, to avoid being influenced by the screen motion. The particle passage is measured at the end of an outlet, which was added to a corresponding experimental screen apparatus for an easier measurement (compare [27]). In each simulation in the investigation here, only one screen surface is used. Before the actual simulations, the desired quantity of particles with the attached amount of water is placed well mixed on a flat surface in the feed bin. At the beginning of each simulation, the flat surface under the particles is removed and the particles drop as bulk material down on the screen surface. While some smaller particles directly pass the apertures with their first attempt, others need more tries or have to stratify through the gaps between larger particles 
343 before reaching the screen surface. After passing the apertures, the particles drop on an

344 inclined wall and move further to the outlet, where size and attached liquid amount as well as

345 the time of passage are recorded and tracked. In this way, the fraction retained per size class

346 over time for various configurations of DEM simulations can be evaluated. An experimental

347 validation of this batch screening process under the influence of moisture can be found in a

348 previous publication by the authors [27].

349 The mechanical and physical particle and wall properties, which are relevant in the simulations,

350 are presented in Table 2. In this investigation, glass spheres are applied in three different 351 equally distributed discrete size classes of $d_{1}=5 \mathrm{~mm}, d_{2}=7 \mathrm{~mm}$, and $d_{3}=10 \mathrm{~mm}$. The 352 particles and the aperture size have the relation $d_{1}<d_{2}<a<d_{3}$. In the following the particle 353 classes are called small $\left(d_{1}\right)$, near mesh $\left(d_{2}\right)$, which has the additional relationship $3540.8 a<d_{2}<a$, and large $\left(d_{3}\right)$. The contact angles between the different materials and water as 355 outlined in Table 2 as well as the respective transfer ratios after a rupture event were obtained 356 in a previous work by the authors [27].

357 Table 2: Mechanical and physical particle and wall properties.

\begin{tabular}{c|c|cc}
\hline & Particles & \multicolumn{2}{|c}{ Walls } \\
\hline Mechanical particle property & Glass & Steel & PVC \\
\hline Diameter $d[\mathrm{~mm}]$ & $5 / 7 / 10 \pm 0.1$ & - & - \\
Mass $m[\mathrm{~g}]$ & $0.1636 / 0.4490 / 1.3090 \pm 0.02$ & - & - \\
Density $\rho\left[\mathrm{kg} / \mathrm{m}^{3}\right]$ & $2.5341 \mathrm{E}+03 \pm 4.10$ & $7.85 \mathrm{E}+03$ & $1.30 \mathrm{E}+03$ \\
Young's modulus $E\left[\mathrm{~N} / \mathrm{m}^{2}\right]$ & $5.00 \mathrm{E}+10$ & $2.08 \mathrm{E}+11$ & $2.20 \mathrm{E}+09$ \\
Poisson's ratio $v[-]$ & 0.2 & 0.30 & 0.4 \\
Stiffness $k_{P P}^{n} / k^{n}{ }_{P W}[\mathrm{~N} / \mathrm{m}]$ & $1.00 \mathrm{E}+05$ & - & - \\
Contact angle $\theta\left[{ }^{\circ}\right]$ & 15 & 45 & 50 \\
\hline
\end{tabular}

The properties for the batch screening DEM simulations can be found in Table 3. The glass spheres are filled into the feed bin with a mass of $m_{p} \approx 1410 \mathrm{~g}$. Three different liquid amounts in the range of $0 \% \leq M \leq 5 \%$ are applied in order to maintain a pendular regime. At the start of each simulation, the walls are dry and each particle holds the same liquid film thickness $P_{l f}$ (compare [24]). The wires in the DEM simulations are approximated as horizontal bars with a semicircular profile. According to the screen applied in the experimental setup, the bars have a diameter of $w=2 \mathrm{~mm}$ resulting in aperture sizes of $a=8 \mathrm{~mm}$.

366 The 3D screen motion is obtained by measurements of an accelerometer ("Sequoia 367 FastTracer PA") fixed under the screen (compare [27]). Initially, the amplitude is set to $368 A=1 \mathrm{~mm}$ resulting in a frequency of approximately $f=50.6 \mathrm{~Hz}$. The motion is elliptical but 369 mainly in vertical direction ( $\mathrm{z}$ - direction of the screen of about $0.9 \mathrm{~mm}$ ) while the motion in $\mathrm{x}$ 370 and $y$ - directions is low with maximum amplitudes of $A<0.1 \mathrm{~mm}$. In the following, the set 
371 amplitude is used to differentiate the cases. In addition to the liquid amount, either the 372 amplitude or the frequency is varied according to the values in Table 3.

373 Table 3: Properties for batch screening DEM simulations.

\begin{tabular}{c|c}
\hline Properties & Values \\
\hline Particle mass $[\mathrm{kg}]$ & $\sim 1.41$ \\
Liquid amount [\%] & $0 / 2.5 / 5$ \\
Surface tension $[\mathrm{N} / \mathrm{m}]$ & 0.07275 \\
Aperture size $[\mathrm{mm}]$ & $8.00 \pm 0.02$ \\
Aperture shape [-] & square \\
Wire diameter [mm] & $2.00 \pm 0.01$ \\
Screen wire profile [-] & semicircular \\
Set amplitude $[\mathrm{mm}]$ & $0.8 / 1 / 1.2$ \\
Frequency [Hz] & 45.8 / 50.6 / 55.4 \\
Stroke behavior & Elliptical, mainly vertical \\
\hline
\end{tabular}

374

375 The DEM parameters coulomb friction $\mu_{c}$, rolling friction $\mu_{\text {roll }}$ and the coefficient of restitution $376 e^{n}$ can be found in Table 4 for contacts of glass spheres with steel and other glass spheres. A 377 detailed strategy to obtain these parameters is outlined in a work by Elskamp et al. [63].

378 Table 4: DEM parameters for glass spheres and various contact partners.

\begin{tabular}{c|c|ccc}
\hline Contact partner 1 & Contact partner 2 & $\mu_{c}[-]$ & $\mu_{\text {roll }}[\mathrm{m}]$ & $e^{n}[-]$ \\
\hline Glass sphere & $\begin{array}{c}\text { Steel (side walls, screen wires, } \\
\text { bottom, outlet walls) } \\
\text { Glass sphere }\end{array}$ & 0.2866 & $1.09 \mathrm{E}-04$ & 0.4351 \\
Glass sphere & 0.1966 & $8.95 \mathrm{E}-05$ & 0.7808 \\
\hline
\end{tabular}

379

380 All parameters for the performed DEM batch screening simulations and the process models 381 are listed in Table 5.

382 Table 5: Overview of parameters for the performed DEM simulations and the process models.

\begin{tabular}{cccc|cccc}
\hline Case No. & $\begin{array}{c}\text { Amplitude } \\
A[\mathrm{~mm}]\end{array}$ & $\begin{array}{c}\text { Frequency } \\
f[\mathrm{~Hz}]\end{array}$ & $\begin{array}{c}\text { Liquid amount } \\
M[\%]\end{array}$ & Case No. & $\begin{array}{c}\text { Amplitude } \\
A[\mathrm{~mm}]\end{array}$ & $\begin{array}{c}\text { Frequency } \\
f[\mathrm{~Hz}]\end{array}$ & $\begin{array}{c}\text { Liquid amount } \\
M[\%]\end{array}$ \\
\hline 1 & 0.8 & 45.8 & 0 & 15 & 1.0 & 55.4 & 2.5 \\
2 & 0.8 & 50.6 & 0 & 16 & 1.2 & 45.8 & 2.5 \\
3 & 0.8 & 55.4 & 0 & 17 & 1.2 & 50.6 & 2.5 \\
4 & 1.0 & 45.8 & 0 & 18 & 1.2 & 55.4 & 2.5 \\
5 & 1.0 & 50.6 & 0 & 19 & 0.8 & 45.8 & 5 \\
6 & 1.0 & 55.4 & 0 & 20 & 0.8 & 50.6 & 5 \\
7 & 1.2 & 45.8 & 0 & 21 & 0.8 & 55.4 & 5 \\
8 & 1.2 & 50.6 & 0 & 22 & 1.0 & 45.8 & 5 \\
9 & 1.2 & 55.4 & 0 & 23 & 1.0 & 50.6 & 5 \\
10 & 0.8 & 45.8 & 2.5 & 24 & 1.0 & 55.4 & 5 \\
11 & 0.8 & 50.6 & 2.5 & 25 & 1.2 & 45.8 & 5 \\
12 & 0.8 & 55.4 & 2.5 & 26 & 1.2 & 50.6 & 5 \\
13 & 1.0 & 45.8 & 2.5 & 27 & 1.2 & 55.4 & 5 \\
14 & 1.0 & 50.6 & 2.5 & & & & 5 \\
\hline
\end{tabular}




\section{Results and discussions}

385 After performing the DEM simulations according to Table 5, the outcome is used to study the 386 fraction retained per size class for different configurations. In addition, the influence of the 387 subprocesses stratification and passage on the screening process is investigated. In the 388 following, parameters of process models are adjusted to fit the DEM screening results and a 389 comparison of the fraction retained per size class between DEM simulations and process 390 models is carried out. As the main novelty, the adjusted parameters are then applied in the 391 process models to predict the fraction retained per size class for various other configurations. 392 Therein, the results of screening processes with operating parameters and liquid amounts 393 between the values of the adjusted cases (interpolation) as well as with larger or lower values 394 (extrapolation) are predicted. Subsequently, the predicted fraction retained values are 395 compared to the results obtained from DEM simulations.

\subsection{Numerical investigations of batch screening under the influence of moisture}

In a first step, only the fraction retained per size class of DEM simulations with various amplitudes, frequencies and liquid amounts are compared. Note that the particles need at least $0.5 \mathrm{~s}$ to reach the end of the outlet resulting in a constant fraction retained until $t=0.5 \mathrm{~s}$. In all simulations (Fig. 3 and Fig. 4), most of the particles pass the apertures in the first 5 seconds, while after $t=5 \mathrm{~s}$ the fraction retained per size class declines only slowly. Additionally, the fraction retained value of the small particles declines faster than the value of near mesh sized particles in all cases. The smaller particles stratify faster through the gaps of the coarse material and pass the apertures more easily. In the first investigations, dry glass spheres with diameters of $d_{1 / 2 / 3}=5 / 7 / 10 \mathrm{~mm}$ are screened with an aperture size of $a=8 \mathrm{~mm}$ (compare Figs. 3a,b). Approximately until $t=4.5 \mathrm{~s}$, the values of the fraction retained of the small and the near mesh sized particles decline fast but nearly unaffected by the screen agitation. In contrast, after $t=4.5 \mathrm{~s}$ obvious influences of the operating parameters are revealed by a different reduction of the fraction retained per size class. The particles in the lower layers are nearly directly able to pass the apertures, whereas the particles of the upper layers have to stratify to the bottom layer, which is intensely influenced by the operating parameters. First, the amplitude is varied from the initial value of $A=1 \mathrm{~mm}$ to $0.8 \mathrm{~mm}$ and $1.2 \mathrm{~mm}$ (Fig. 3a). In the initial case, approximately $80 \%$ of the near mesh sized and $87 \%$ of the small particles have already passed the apertures at $t=5 \mathrm{~s}$. At $t=10 \mathrm{~s}$ only $10 \%$ and $1.5 \%$ of near mesh and small particles remain on the screen, respectively. After $t=15 \mathrm{~s}$, nearly all undersized particles were able to pass through the apertures. 





d
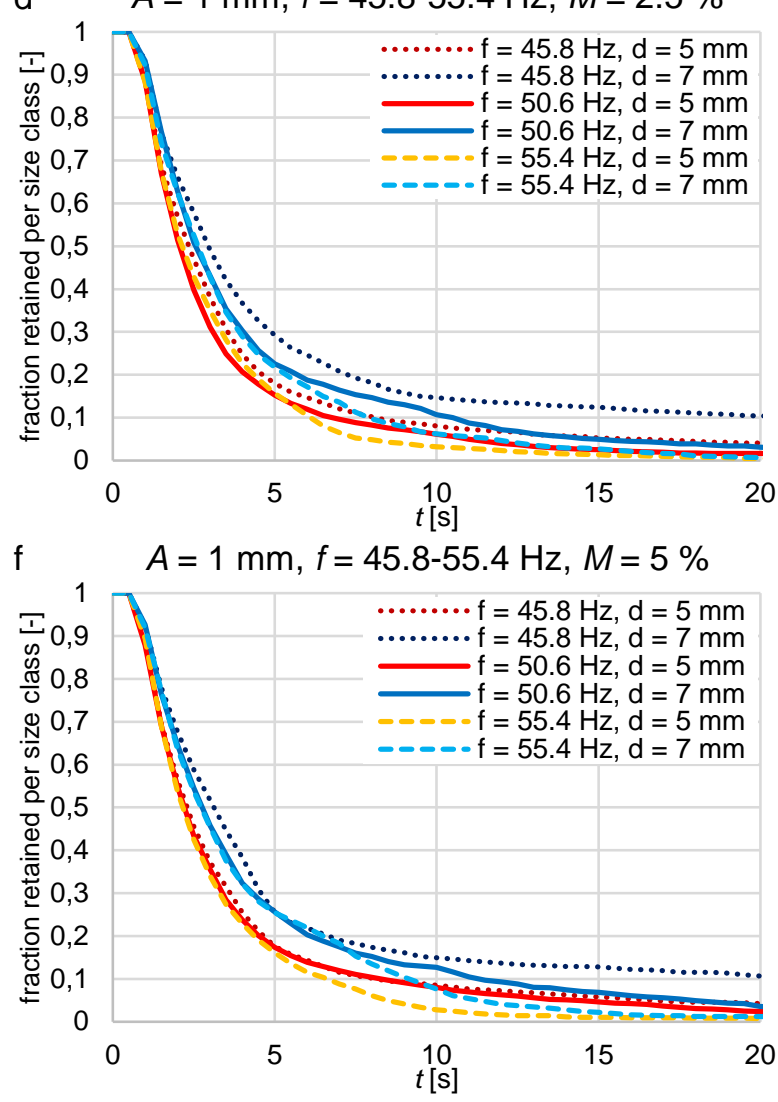

h $\quad A=0.8-1.2 \mathrm{~mm}, f=45.8-55.4 \mathrm{~Hz}, M=5 \%$

g $\quad A=0.8-1.2 \mathrm{~mm}, f=55.4-45.8 \mathrm{~Hz}, M=5 \%$
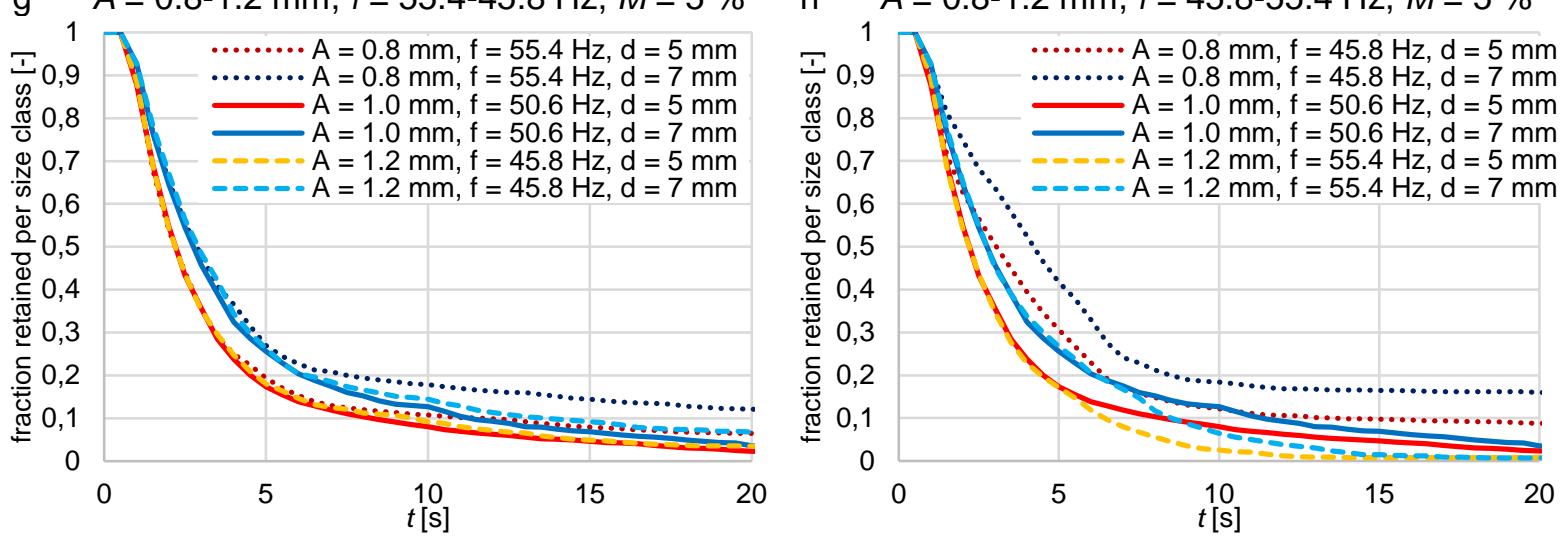

Fig. 3: Fraction retained on the screen over time presented for the small $\left(d_{3}=5 \mathrm{~mm}\right)$ and near mesh sized particle fractions $\left(d_{2}=7 \mathrm{~mm}\right)$ applying $(\mathrm{a}, \mathrm{b}) \mathrm{dry}(M=0 \%)$ and $(\mathrm{c}-\mathrm{h})$ wet glass spheres $(M=2.5 / 5 \%)$ with $\mathrm{a}=8 \mathrm{~mm}\left(d_{1 / 2 / 3}=5 / 7 / 10 \mathrm{~mm}\right)$ and $(\mathrm{a}, \mathrm{c}, \mathrm{e})$ a variation in the amplitude, $(b, d, f)$ a variation in the frequency and $(g, h)$ a variation in the amplitude and the frequency. All results are obtained by DEM simulations. 
422 When increasing the amplitude to $A=1.2 \mathrm{~mm}$, the fraction retained values for both size 423 classes decline faster between $t=5 \mathrm{~s}$ and $t=10 \mathrm{~s}$, resulting in an earlier depletion of the small 424 particles. However, the near mesh sized particles need about the same time as before under 425 the influence of the initial amplitude. By applying a larger stroke length, the stratification for the 426 small particles due to a loosening of the layers seems to be supported. A decrease of the amplitude to $A=0.8 \mathrm{~mm}$ leads to a reduced decline of the fraction retained values for both size classes after $t=5 \mathrm{~s}$ and to an appreciable amount of particles remaining on the screen after $t=20 \mathrm{~s}$. The length of the stroke is not long enough to clear the apertures from pegging particles and to give the smaller particles enough possibilities to stratify through the larger ones

431 to the screen surface.

432 Thereafter, the amplitude is kept constant and the initial frequency of $f=50.6 \mathrm{~Hz}$ is changed to $45.8 \mathrm{~Hz}$ and $55.4 \mathrm{~Hz}$ (Fig. 3b). Besides the earlier depletion of the near mesh sized particles, an increase of the frequency to $f=55.4 \mathrm{~Hz}$ results in nearly the same intensification of the inclination like an increase of the amplitude to $A=1.2 \mathrm{~mm}$ due to the same reason. In contrast, a reduction of the frequency to $f=45.8 \mathrm{~Hz}$ has a lower impact than a decrease of the amplitude to $A=0.8 \mathrm{~mm}$. Nevertheless, some of the particles remain on the screen after $t=20 \mathrm{~s}$. The intensity of the stroke is too low to induce large throws of the coarse material to build up gaps for the small particles to pass through them.

In the next investigations, the liquid amount is increased from $M=0 \%$ to $M=2.5 \%$ (Figs. 3c,d) and $M=5 \%$ (Figs. 3e,f), including variations in the amplitude and the frequency equivalent to those for dry screening. The qualitative results are similar to those with dry particles. A larger amplitude slightly increases the decline of the fraction retained while a larger frequency has a stronger decreasing influence on this value. In contrast, lower amplitudes and frequencies reduce the particle passage and thereby the decline of the fraction retained per size class. In addition, several particles remain on the screen after $t=20 \mathrm{~s}$.

The influence of changes in both operating parameters (amplitude and frequency) with a liquid amount of $M=5 \%$ is shown in Figs. $3 \mathrm{~g}$,h. For an increase in amplitude and a decrease in frequency, the simulation results are very similar to the initial ones, whereas a larger frequency is not able to compensate for the influence of a lower amplitude when a liquid amount of $M=$ $5 \%$ is applied (compare Fig. $3 g$ ). Although the frequency is enlarged, a low amplitude under moist conditions leads to short strokes, which are not able to provide enough opportunities for the undersized particles to stratify towards the apertures to pass through them. In Fig. 3h, under the influence of $M=5 \%$, the operating parameters are both increased or both decreased, respectively. As expected, these simulation results reveal the fastest and the slowest decrease of the fraction retained when applying the same liquid amount. 

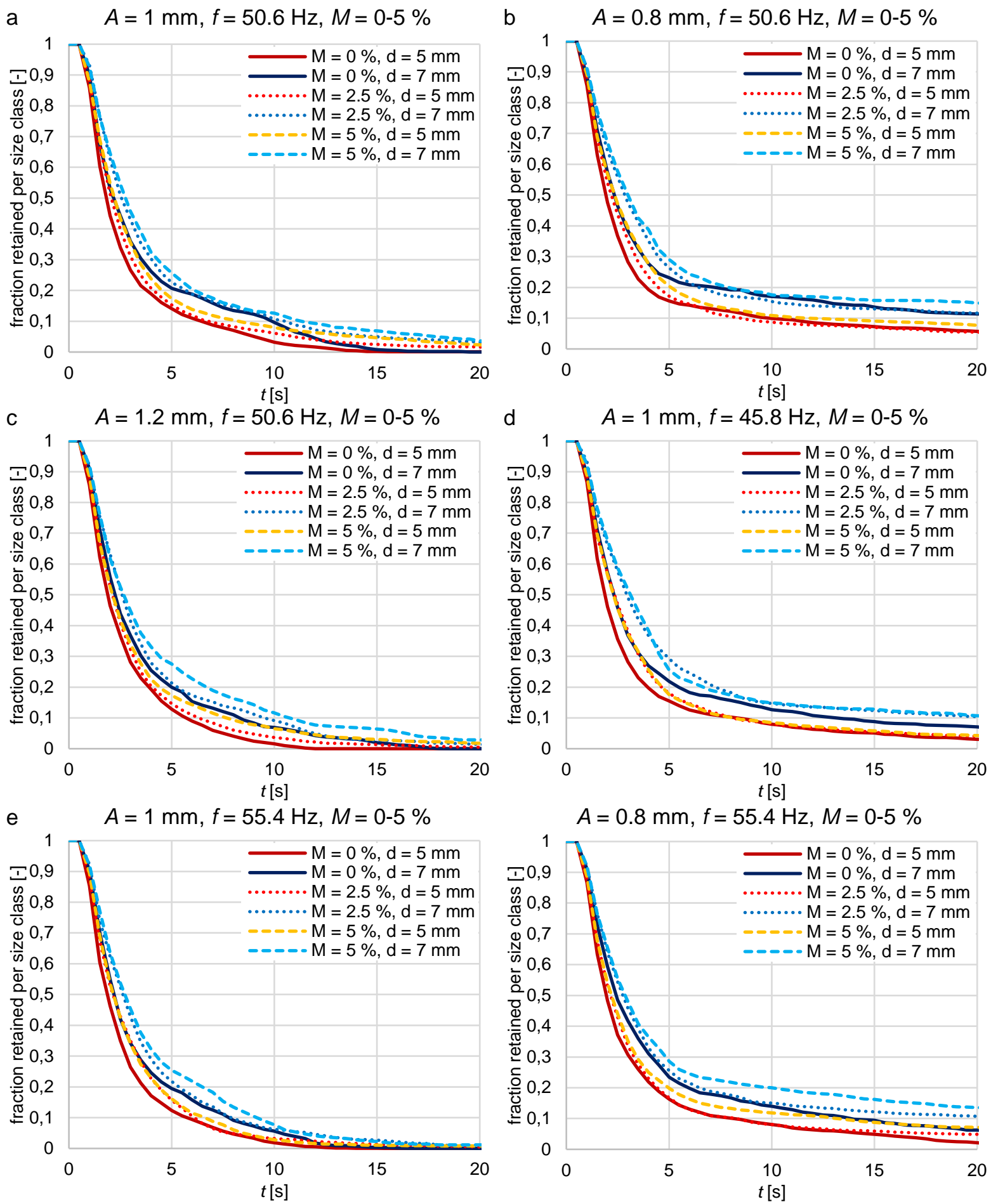

Fig. 4: Fraction retained on the screen over time presented for the small $\left(d_{3}=5 \mathrm{~mm}\right)$ and near mesh sized particle fractions $\left(d_{2}=7 \mathrm{~mm}\right)$ applying glass spheres with a varying amount of liquid $(M=0 / 2.5 / 5 \%)$ with $\mathrm{a}=8 \mathrm{~mm}\left(d_{1 / 2 / 3}=5 / 7 / 10 \mathrm{~mm}\right)$ and $(\mathrm{a})$ initially $A=1 \mathrm{~mm}$ and $f=50.6 \mathrm{~Hz}$. The amplitude is changed to (b) $A=0.8 \mathrm{~mm}$ and (c) $A=1.2 \mathrm{~mm}$ as well as the frequency to (d) $f=45.8 \mathrm{~Hz}$ and (e) $f=55.4 \mathrm{~Hz}$. The amplitude and frequency are changed to (f) $A=0.8 \mathrm{~mm}$ and $f=55.4 \mathrm{~Hz}$. All results are obtained by DEM simulations.

462 In order to find out the influence of the added water, simulations with various liquid amounts 463 and constant operating parameters were performed and are shown in Fig. 4. For the initial configuration with $A=1 \mathrm{~mm}$ and $f=50.6 \mathrm{~Hz}$ (Fig. 4a), an increase of the liquid amount results in a lower passage rate and thereby to larger values for the fraction retained per size class 466 independent of particle size. After $t=20 \mathrm{~s}$, some small and near mesh moist particles are still 

on the screen surface. By adding a small liquid amount, the particles stick to each other and the loosening of the particle layers and thereby the stratification is reduced.

In the next investigations, one operating parameter is changed in each case in comparison to the initial configuration. The results for the simulations with a lower amplitude of $A=0.8 \mathrm{~mm}$ (Fig. 4b) reveal a similar impact of the added water, but after $t=5 \mathrm{~s}$ the curves for the dry particles stagnate more intensively than the other curves due to a larger amount of blocked apertures and the difference to the configuration with $M=2.5 \%$ is equalized. If the amplitude is increased to $A=1.2 \mathrm{~mm}$ (Fig. 4c), the influence of the liquid amount is similar to the initial case. The fraction retained is larger if more water is in the system and at $t=20 \mathrm{~s}$, some of the moist undersized particles are still on the screen.

The results for a lower frequency of $f=45.8 \mathrm{~Hz}$ (Fig. 4d) reveal an equivalent difference between the cases under dry and slightly moist conditions $(M=2.5 \%)$ as in the initial configuration. However, a larger liquid amount has only a negligible influence on the fraction retained per size class. If the frequency is increased to $f=55.4 \mathrm{~Hz}$ (Fig. 4e), the passage rate is slowed down for a larger liquid amount and hence, the fraction retained per size class is larger as in the initial configuration. In contrast, the differences between the small particles under the influence of various liquid amounts $(M=2.5 / 5 \%)$ are marginal and all particles are screened after $t=20 \mathrm{~s}$. For a lower amplitude of $A=0.8 \mathrm{~mm}$ and a larger frequency of $f=55.4 \mathrm{~Hz}$ (Fig. 4f), the decrease of the fraction retained is slightly lower for $M=2.5 \%$ in comparison to dry screening, but obviously lower for $M=5 \%$. In the dry case, the larger frequency is able to compensate for the low amplitude, which is not possible for a larger liquid amount.

\subsection{Stratification and passage under the influence of moisture}

By analyzing the previous results, it is only possible to compare the passage combined with the stratification. In the following, both subprocesses are studied separately to figure out the relevance of considering them individually in a process model for batch screening.

From a previous work by the authors [44], it is already known that larger amplitudes and frequencies can lead to a faster stratification up to a critical value. That statement has to be verified for the setup applied in this investigation. Additionally, the influence of the liquid amount on the stratification is studied. In order to evaluate the particle stratification, several possibilities are available (compare $[8,44])$.

For the first one, the already performed simulations are sufficient. Therein, the particle bed in the screening process is divided in the bottom layer (compare section 3 ) and the particles above the bottom layer (top layer). The average time, which the undersized particles need to 
stratify from the top layer to the bottom layer, is compared in Fig. 5 for the near mesh sized $\left(t_{d 2}\right)$ and small particles $\left(t_{d 3}\right)$. In all the simulations, the particles with the larger diameter $d_{2}$

503

504

505

506

507

508

509

510

511

512

513

514

515

516

517

$$
h^{*}=\sum_{j=1}^{n_{\text {class }}}\left|\left(\sum_{i=1}^{n_{\text {part }, j}} z_{i, j} / n_{\text {part }, j}\right)-\left(\sum_{i=1}^{n_{\text {part }}} z_{i} / n_{\text {part }}\right)\right|,
$$

518 where $n_{\text {class }}$ is the number of size classes, $n_{\text {part }}$ is the total number of particles, $n_{\text {part, }}$ is the 519 number of particles in the respective size class $j, z_{i}$ is the height of a particle $i$ and $z_{i, j}$ is the 520 height of particle $i$ belonging to size class $j$ in the system. In Fig. 6 the accumulated deviation $521 h^{*}$ over time $t$ is shown for the initial case $(A=1 \mathrm{~mm}, f=50.6 \mathrm{~Hz}, \mathrm{M}=0 \%)$ and different amplitudes, frequencies and liquid amounts, where only one parameter is varied at a time.

523 At $t=0 \mathrm{~s}$, the particles are well mixed resulting in $h^{*}=0$, whereas this value increases for a 524 progressive stratification. The results confirm the observations made before. A larger 

$h^{\star}$.

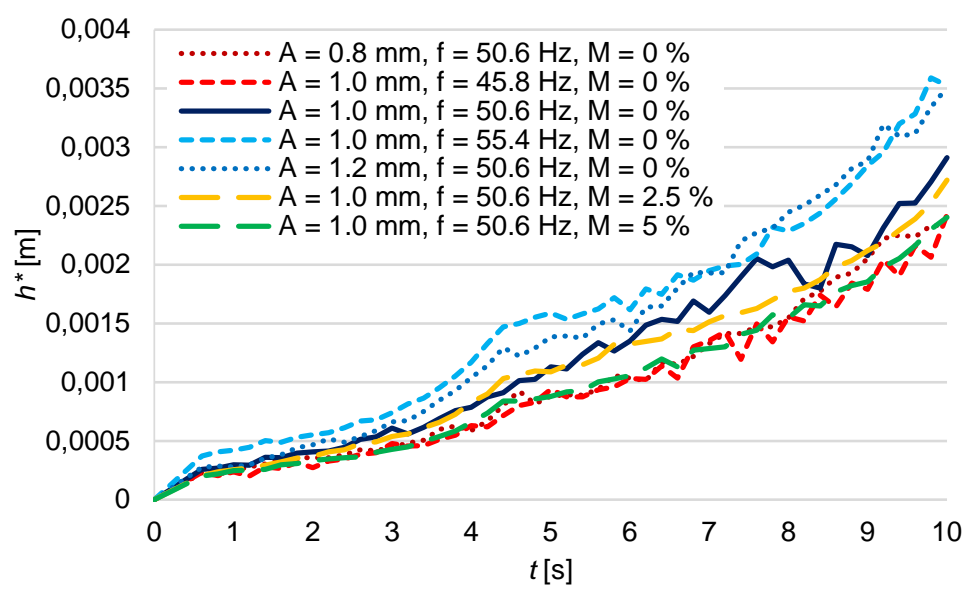

Fig. 6: Accumulated deviation of the average particle height per size class from the total average particle height $h^{\star}$ for different amplitudes, frequencies and liquid amounts in comparison to the initial configuration $(A=1 \mathrm{~mm}, f=50.6 \mathrm{~Hz}, \mathrm{M}=0 \%)$ over time $t$ in the batch apparatus without apertures $(a=0)$. Only one parameter is varied in each simulation.

Concerning the subprocess passage, it is known from the work by Dong et al. [45], that larger amplitudes and frequencies result in less attempts for the particles to pass the apertures. This is only valid for a screening process with a thin layer of particles. Therefore, the initial setup is applied, but only a quarter of the particles is directly placed above the screen surface. The passage of a particle is recorded as soon as it is tracked below the screen surface. The simulations are compared by the fraction retained per size class over a time period of $5 \mathrm{~s}$ and their results are presented in Fig. 7. The operating parameters and the liquid amount are changed according to Table 3 , but only one parameter is varied at a time.
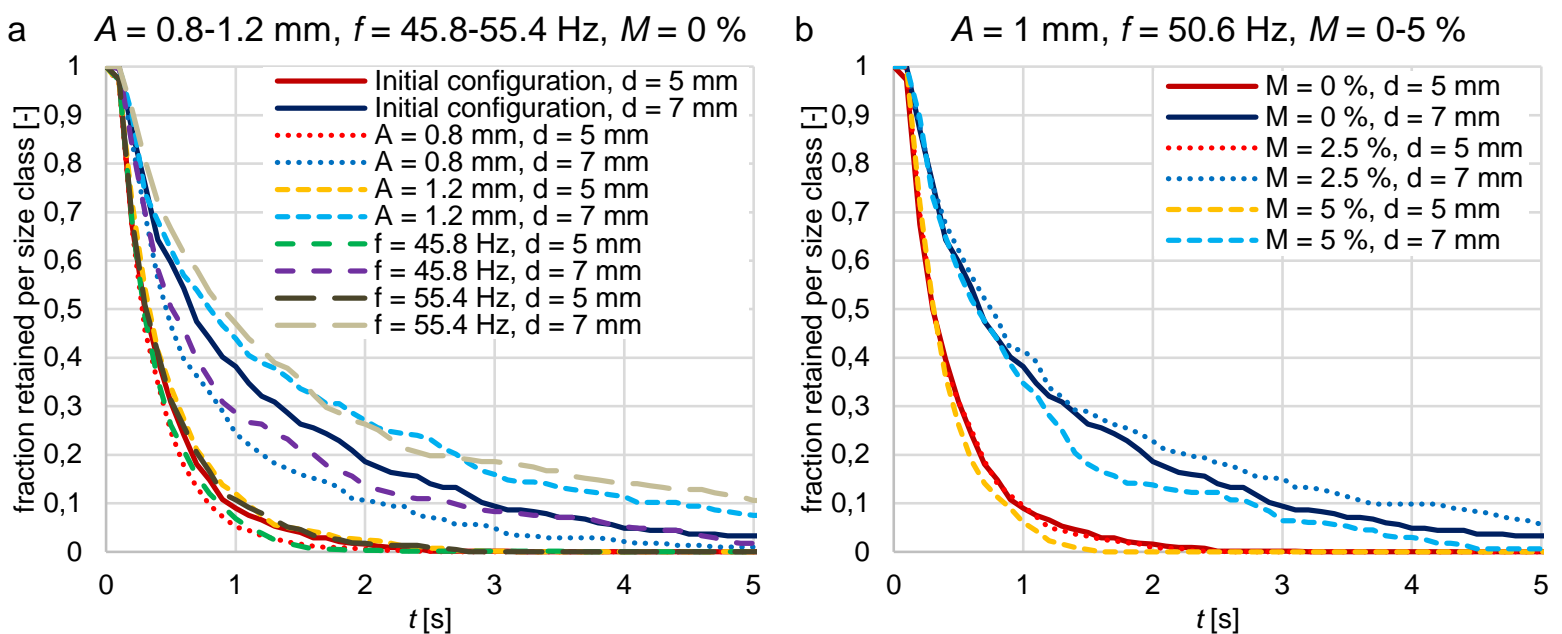

Fig. 7: Fraction retained on the screen over time presented for the small $\left(d_{3}=5 \mathrm{~mm}\right)$ and near mesh sized particle fractions $\left(d_{2}=7 \mathrm{~mm}\right)$ applying a thin layer of glass spheres with $a=8 \mathrm{~mm}\left(d_{1 / 2 / 3}=5 / 7 / 10 \mathrm{~mm}\right)$ and initially $A=1 \mathrm{~mm}, f=50.6 \mathrm{~Hz}$ and $M=0 \%$. This initial case is compared to simulations where either (a) the amplitude or frequency or (b) the liquid amount is varied according to Table 3.

544 Particularly for the near mesh sized particles, but also for the small particles, the fraction retained decreases faster for a lower amplitude or frequency and vice versa (compare Fig. 7a). 
546 The particle throws are shorter and thereby, the particles get more attempts to pass the 547 apertures. In Fig. $7 b$ the liquid amount is increased from $M=0 \%$ to $2.5 \%$ and $5 \%$. The 548 passage of the small particles is slightly increased for a larger liquid amount, whereas the near 549 mesh sized particles reveal varying results. While an increase to $M=2.5 \%$ slows down the 550 passage, the fraction retained value decreases faster for a larger liquid amount of $M=5 \%$.

551 The reason for this varying behavior is that the throws of the particles under the influence of 552 liquid are shorter giving the particles more attempts to pass the apertures, whereas the liquid 553 bridges between the particles and the screen wires aggravate the passage.

554 In summary, the amplitude, the frequency and the liquid amount influence the two 555 subprocesses stratification and passage contrarily to some extent. Therefore, these processes 556 should be considered separately in a process model, which is further discussed in the following 557 section.

5.3 Application of extended process models for batch screening under the 559 influence of moisture

560 In this section, the three introduced modified process models for batch screening under the 561 influence of moisture are compared by adjusting their parameters to fit the results obtained by 562 DEM simulations. Thereby, the model results are first adjusted with one set of parameters to 563 all DEM simulation results, giving information about the quality of representing a broad range 564 of various batch screening configurations. In order to quantify these results, the obtained 565 deviations are compared with the lowest possible deviations that could be obtained when 566 applying one set of parameters for each configuration in the process models. Thereby, the 567 deviations of one case are only dependent on the accuracy of the model equation in 568 representing the progression of the fraction retained of this case. Subsequently, the capability 569 of the process models to predict results of batch screening under dry and moist conditions is 570 investigated for the first time. Therein, the adjusted parameters are applied to predict screening 571 results with operating parameters or liquid amounts between the adjusted ones (interpolation) 572 and with lower or larger values (extrapolation), which could be relevant for quantifying the 573 feasibility in industrial applications.

574 First, one set of adjustable parameters of the process models are adjusted to best fit the results 575 of the fraction retained per size class of all investigated simulations according to Table 5. In 576 Fig. 8, some examples of the progression of the fraction retained per size class until $t=20 \mathrm{~s}$ 577 for the initial case (Fig. 8a), two different liquid amounts (Figs. 8b,c), a larger frequency 578 (Fig. 8d), a larger amplitude combined with a small liquid amount (Figs. 8c,e) as well as a large 

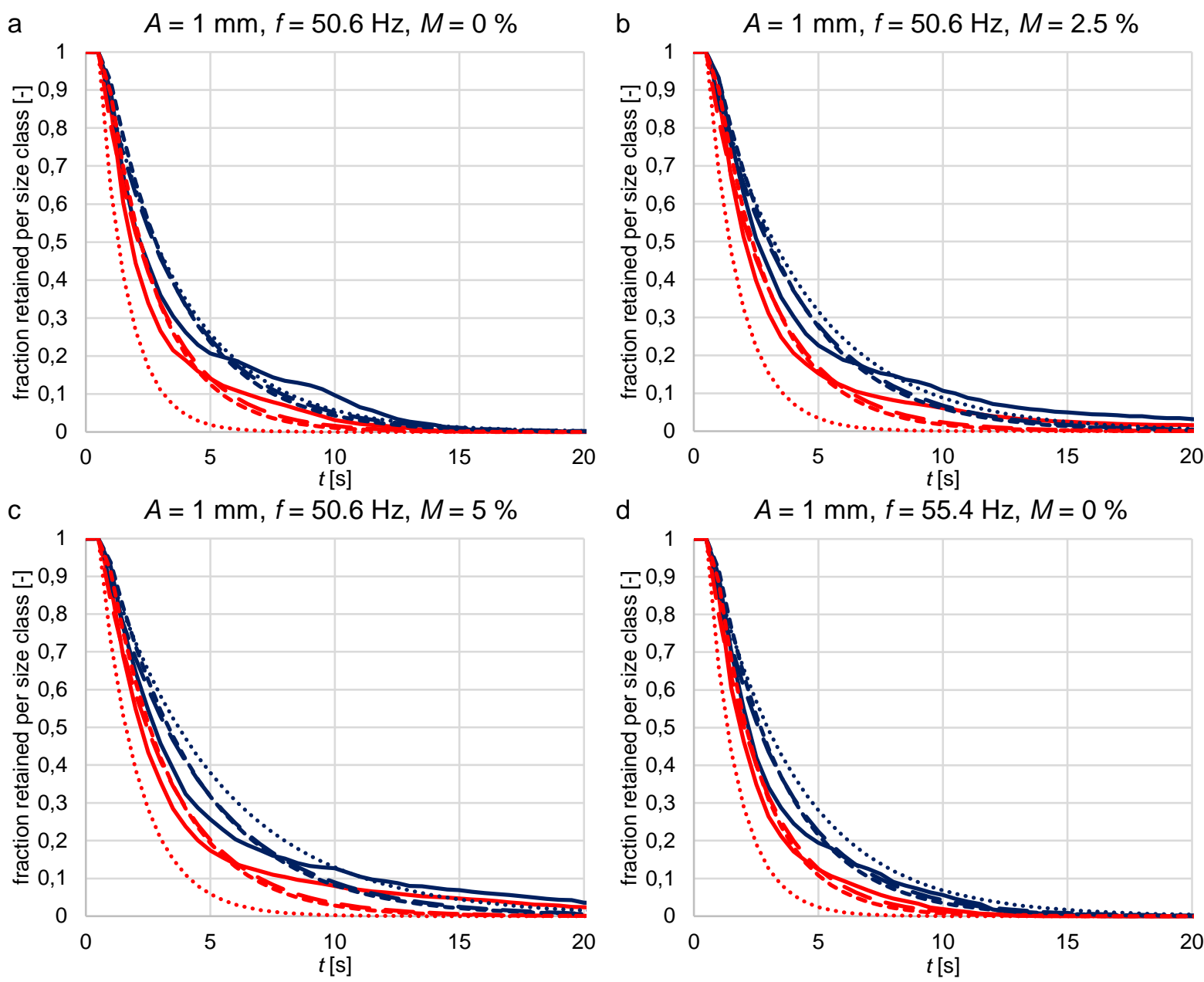

d

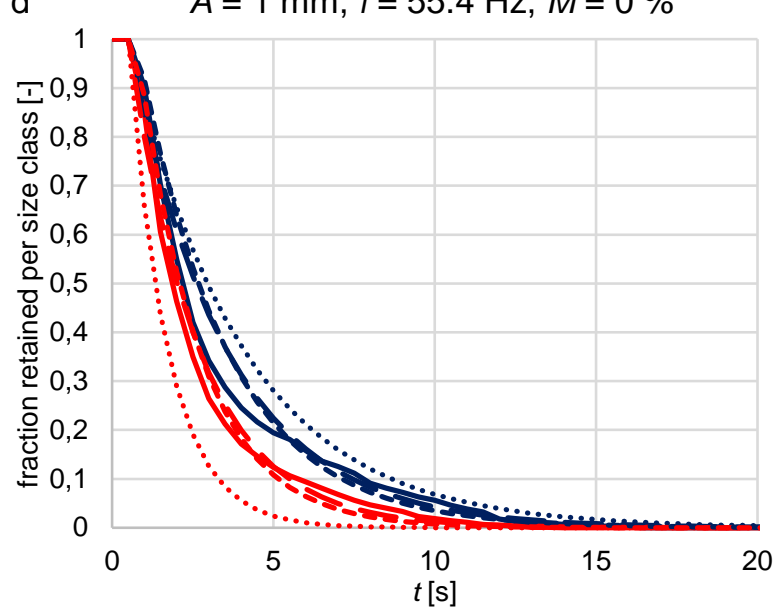

e $\quad A=1.2 \mathrm{~mm}, f=50.6 \mathrm{~Hz}, M=2.5 \%$
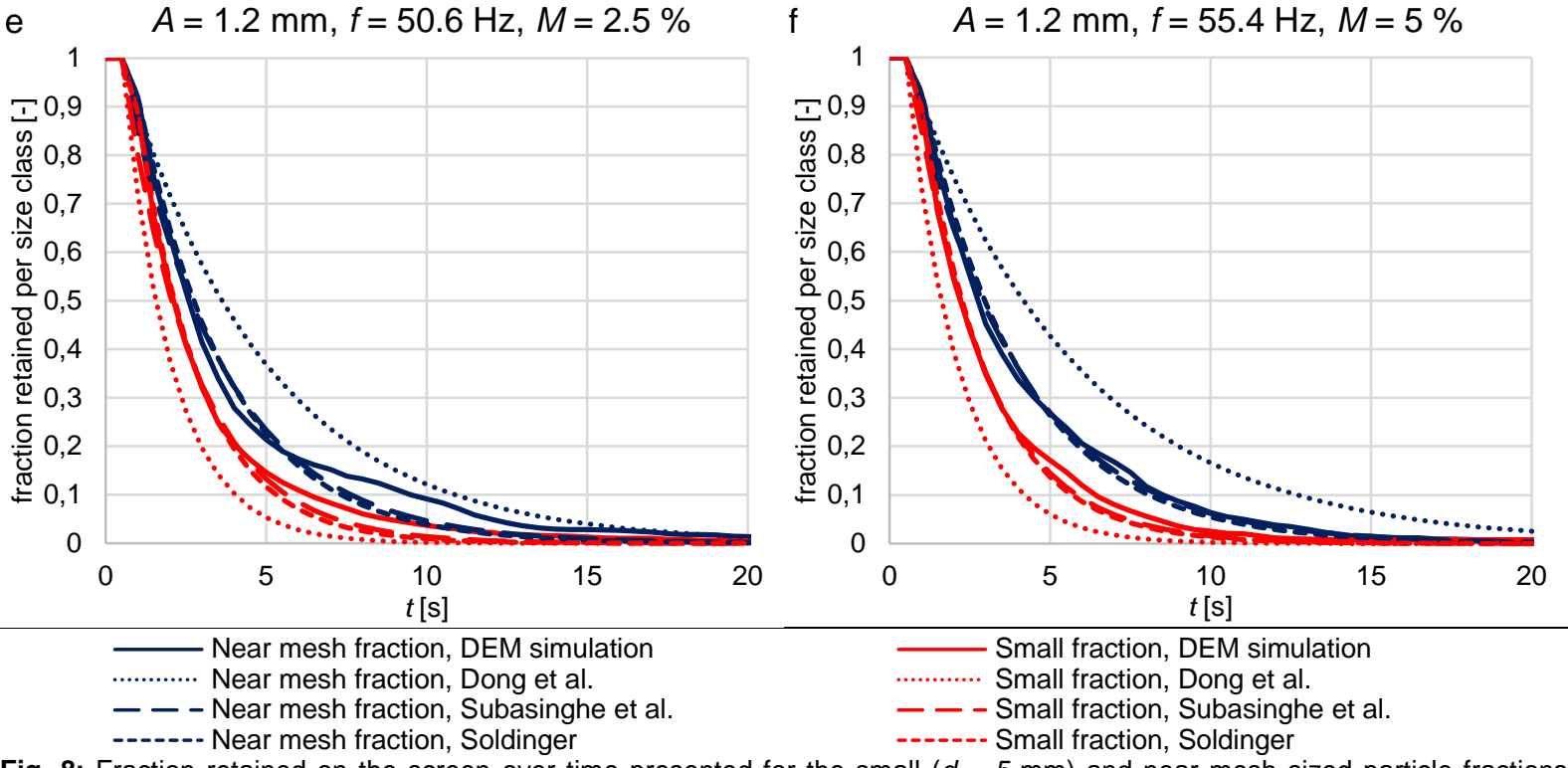

$t[\mathrm{~s}]$

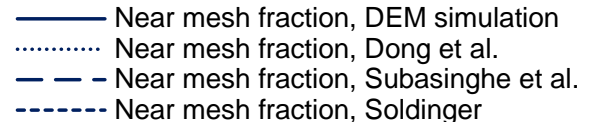

-...-. Small fraction, Soldinger

Fig. 8: Fraction retained on the screen over time presented for the small $\left(d_{3}=5 \mathrm{~mm}\right)$ and near mesh sized particle fractions $\left(d_{2}=7 \mathrm{~mm}\right)$ for various phenomenological screening process models and the DEM simulations applying glass spheres with $a=8 \mathrm{~mm}\left(d_{1 / 2 / 3}=5 / 7 / 10 \mathrm{~mm}\right)$. Results are obtained for (a) $A=1 \mathrm{~mm}, f=50.6 \mathrm{~Hz}$ and $M=0 \%$, (b) $A=1 \mathrm{~mm}, f=50.6 \mathrm{~Hz}$ and $M=2.5 \%$, (c) $A=1 \mathrm{~mm}, f=50.6 \mathrm{~Hz}$ and $M=5 \%$, (d) $A=1 \mathrm{~mm}, f=55.4 \mathrm{~Hz}$ and $M=0 \%$, (e) $A=1.2 \mathrm{~mm}, f=50.6 \mathrm{~Hz}$ and $M=0 \%$ as well as for (f) $A=1.2 \mathrm{~mm}, f=55.4 \mathrm{~Hz}$ and $M=5 \%$. 
586 It is obvious, that model No. 1 underpredicts the fraction retained of the small particles and 587 mostly overpredicts the fraction retained of the near mesh particles for all configurations shown 588 here. The other models reveal only slight deviations, whereby the results of model No. 2 are 589 a bit closer to the simulation results. A finding of section 5.1 was that a lower amount of liquid 590 as well as a more intense screen motion support the decline of the fraction retained and that 591 smaller particles pass the apertures faster than the near mesh sized particles. These facts are 592 qualitatively represented by the models No. 2 and No. 3 in all cases and by model No. 1 in 593 most cases (compare Fig. 8).

594 For a larger frequency (Fig. 8d) or amplitude (Fig. 8e) or both (Fig. 8f) in comparison with the 595 initial operating parameters (Figs. 8a,b), model No. 1 obtains a slightly larger value for the 596 fraction retained per size class. Due to being adjusted with one set of parameters to all 597 simulation results and not considering the stratification process, model No. 1 is not able to 598 compensate the delaying influence on the passage by the supporting impacts on the 599 stratification when larger operating parameters are applied. Conversely, the deviations for 600 configurations with a larger amount of liquid would be more intense, if the results of this model 601 are fitted more accurately to cases with larger operating parameters. Both other models 602 consider the stratification and the passage with different adjustable parameters and therefore, 603 they are able to balance the contrasting effects and to provide results close to the simulated 604 ones. However, it should be noted, that these better results are accompanied by applying twice 605 the number of adjustable parameters.

606 To benchmark the introduced models over a larger number of investigations, an average 607 deviation of the fraction retained per size class obtained by DEM simulations and process 608 models is calculated. For the different undersized particle classes $i$, the average of the obtained 609 fractional deviations is given by $\left(\sum_{i=1}^{l}\left(\sum_{k=1}^{j}\left|Y_{\text {mod }}(i, k)-Y_{\text {sim }}(i, k)\right|\right)\right) /(j \cdot r)$, where $j$ is the total 610 number of considered time steps $k$ and $r$ is the total number of undersized fractions (here $611 r=2)$. Note that the time of the screening process $t=20 \mathrm{~s}$ is divided into intervals of $\Delta t=0.5 \mathrm{~s}$.

612 The averaged deviations between the fraction retained per size class obtained by 613 phenomenological screening models sorted according to Table 1 and discrete element 614 simulations summed up for all investigated simulations according to Table 5 are presented in 615 Fig. 9 and Fig. 10. In Fig. 9, the process models are adjusted to the simulation results applying 616 one set of parameters for all simulations (bars left of each model number referred to as "left 617 bars" in the following). The average deviations for the models are 0.0593 (No. 1), 0.0387 618 (No. 2) and 0.0417 (No. 3). Therein, the parameter sets for the three models are as follows: 
- No. 1: $k=1142.6444, \alpha=-0.0193, y=-161.2886$

620

621

622

623

624

625

626

627

628

- No. $2: k_{s}=1.3883, \alpha=0.8288, \gamma=2.2026, k_{p}=0.0542, \beta=-5.5913, \delta=0.4581$

- No. 3: $k_{s}=5.3935, \alpha=-9.3318, \gamma=9.6818, k_{p}=5.0896, \beta=0.7160, \delta=2.4247$

Additionally, these results are compared to the best possible adjustment when one set of parameters for each simulation is used (Fig. 9, bars on the right side of each model number referred to as "right bars" in the following; parameter sets not shown here). Here, the best possible average deviations for the models are 0.0392 (No. 1 / No. 2) and 0.0403 (No. 3). The deviations are very similar for all three models, because the quality of the adjustment in comparison to the other models is independent of the values of the operating parameters or the liquid amount (compare Fig. 9, right bars).

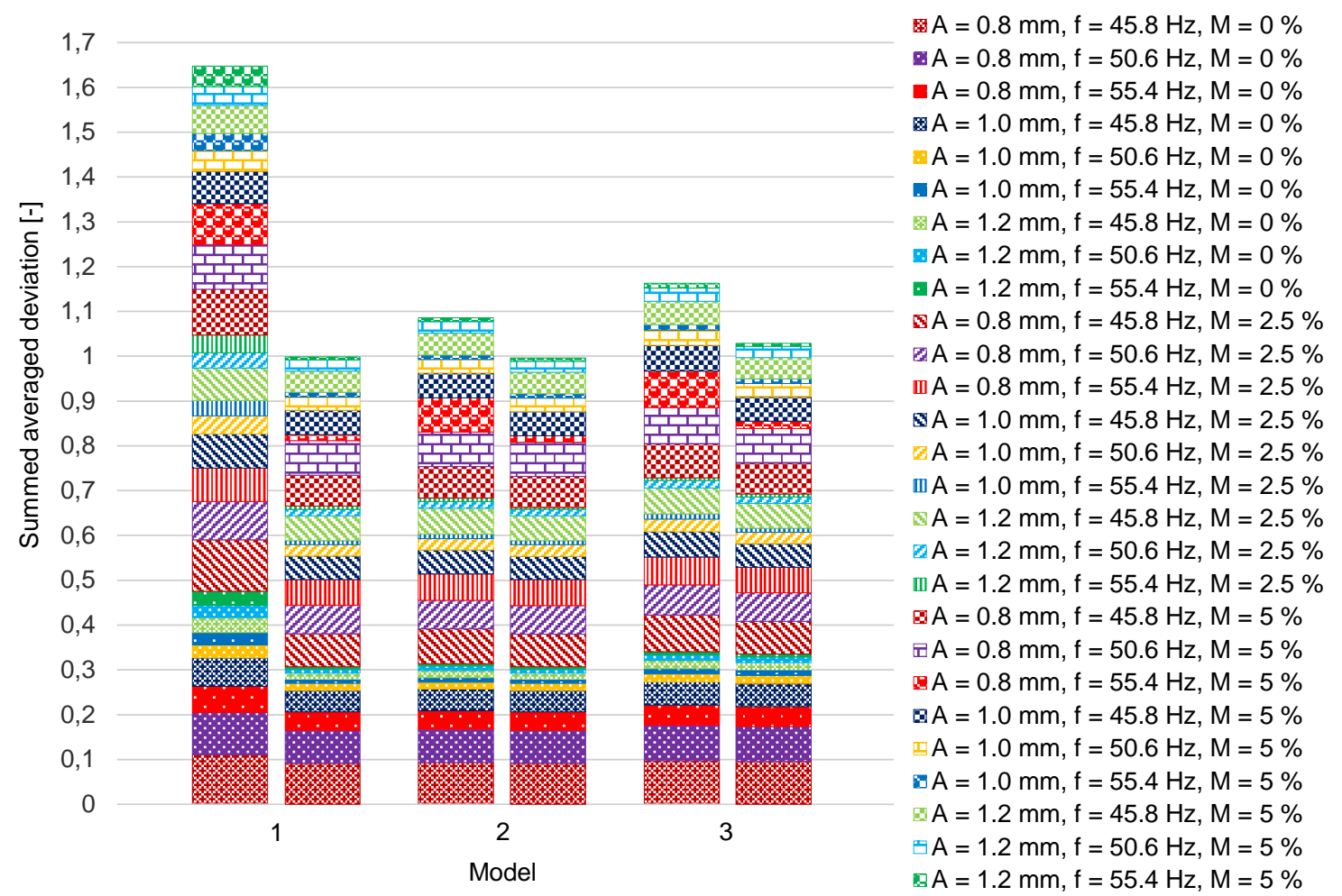

629

630

631

632

633

634

635

636

637

638

639

640

641

Fig. 9: Deviations between the fraction retained per size class obtained by phenomenological screening models sorted according to Table 1 and discrete element simulations summed up for all investigated simulations according to Table 5 . The process models are adjusted to the simulation results by applying one set of parameters for all simulations (bars on the left side of each model number) and by applying one set of parameters for each simulation, showing the best possible adjustment (bars on the right side of each model number).

Overall, all models are better in representing simulations with a faster decline of the fraction retained, whereas the deviations increase for a slower decline of the fraction retained due to lower amplitudes, frequencies and larger liquid amounts. If the models are adjusted with one set of parameters to all simulation results (compare Fig. 9, left bars), the ranking of the models from the lowest to the largest deviation is No. 2, No. 3 and No. 1, for each configuration. Thereby, the results for the models No. 2 and No. 3 are similar whether one set of parameters is used for all simulations (Fig. 9, left bars) or one for each simulation (Fig. 9, right bars), which represents the best possible adjustment for the respective model. In contrast, the deviations 
642 for model No. 1 are much larger if only one set of parameters is applied. It can be concluded, 643 that the functional forms of all three models are able to represent the progression of the fraction 644 retained per size class for one individual configuration well. However, in batch screening with 645 several particle layers, it is essential to consider the subprocesses stratification and passage 646 like in model No. 2 and No. 3 to represent the results of a wider range of simulation 647 configurations.

648 The capability of the extended process models to predict simulation results by applying one 649 set of parameters adjusted to simulation results under different configurations is investigated 650 in the following and the summed averaged deviation is shown in Fig. 10. Note that only the 651 deviations of the predicted results to the DEM simulations and not those of the adjusted ones 652 are compared. In this analysis, the process models are adjusted to the first named simulations 653 (numbers in front of the arrows in Fig. 10) before the adjusted parameter set is applied to 654 predict the second named simulations (numbers behind the arrows in Fig. 10). The ranking of 655 the models summed up for all configurations is again No. 2, No. 3 and No. 1, but the individual deviations reveal some interesting particularities. In Figs. 10a-c, only one value of the operating parameters or the liquid amount is varied, while the others are kept constant on the initial, the lower or the larger value. Thereby, the red, blue and green parts of the bars indicate a change in the liquid amount, the amplitude or the frequency, respectively.

In Fig. 10a, the value of one operating parameter (indicated as blue (amplitude) and green bars (frequency)) or the liquid amount (indicated as red bars) of one predicted result is located between the values of two adjusted ones (e.g. $M=0 \% / M=5 \% \rightarrow M=2.5 \%$ ). The results reveal that all models are able to predict these interpolated values for the fraction retained per size class very well. If only the liquid amount is varied and the operating parameters are kept constant, the results for the models Nos. 1 and 2 are equal. The functional forms of both models are able to predict these results well, because the fraction retained values of the respective predicted configuration are located mostly between those of the adjusted ones. In contrast, model No. 1 reveals more deviations when simulation results with different operating parameters should be predicted. Under these conditions, nearly the same results are obtained by applying the models Nos. 2 and 3, because they both consider the subprocesses stratification and passage, which are influenced contrarily by the operating parameters. In Fig. 10b, the values of the liquid amount or of the operating parameters of two adjusted results are larger than the value of one predicted configuration (e.g. $M=2.5 \% / M=5 \% \rightarrow$

$674 M=0 \%)$. These extrapolated results reveal overall more deviations than in the investigation 675 before, although the outcomes with different liquid amounts are predicted more precisely and 676 nearly independent of the applied model. Larger deviations occur when the result of one 
677 simulation with a low amplitude or frequency should be predicted. The process models can 678 predict the outcome of the configurations with larger operating parameters with a fast decline 679 in the fraction retained value very well. As a negative consequence, particularly model No. 1 680 fails to satisfactorily predict simulation results obtained with lower operating parameters, which 681 are nearly stagnating.

a

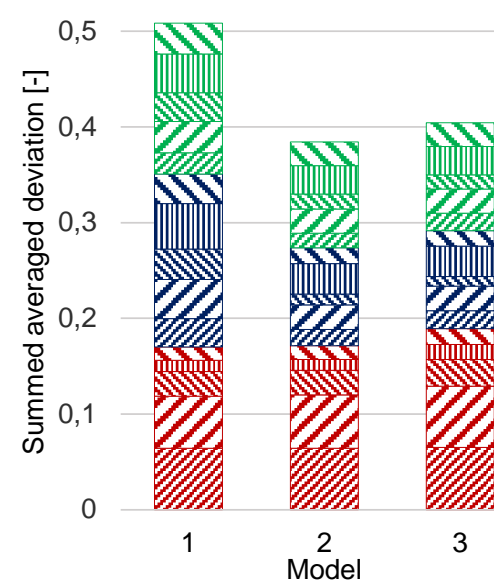

C

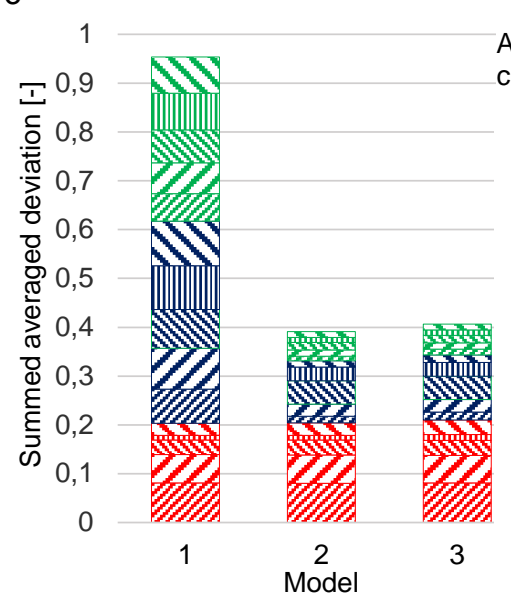

b

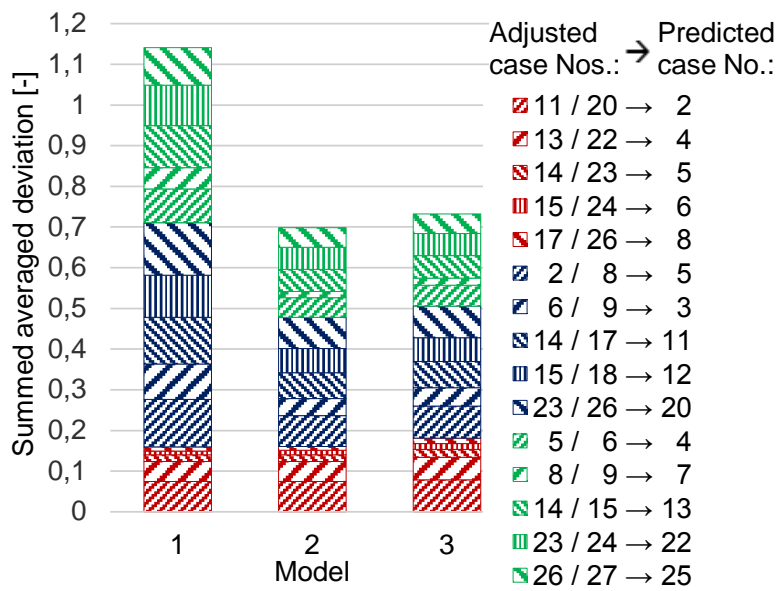

d

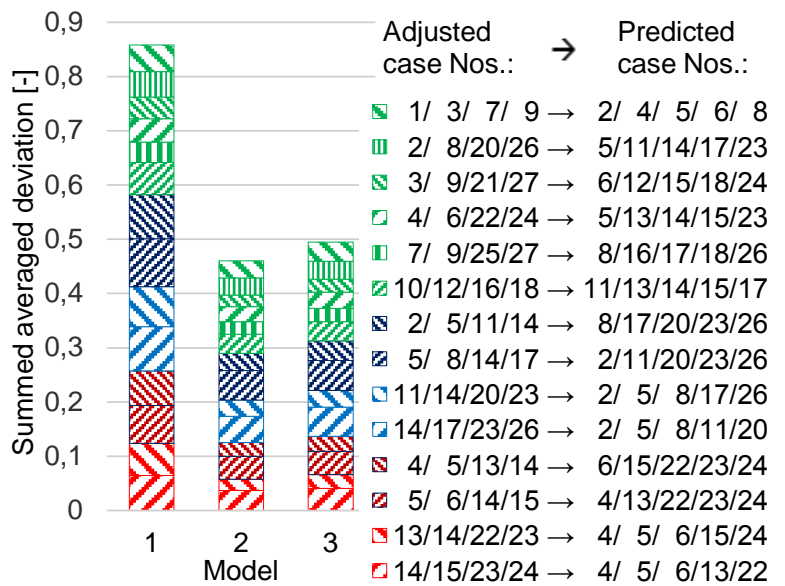

682

683

684

685

686

687

688

689

690

691

692

693

694

Fig. 10: Summed up deviations between the fraction retained per size class obtained predictively by the process models sorted according to Table 1 and DEM simulations for various configurations according to Table 5 . The process models are adjusted to the first named simulations (numbers in front of the arrow) before the adjusted parameters are applied to predict the second named simulations (numbers behind the arrow). The amplitude, frequency or liquid amount of one predicted result is (a) located between (interpolation), (b) lower than or (c) larger than the value of two adjusted ones (extrapolation). (d) Five predicted results are obtained with one set of parameters of four adjusted cases.

The results of predicting the fraction retained per size class for configurations with larger operating parameters or liquid amounts (e.g. $M=0 \% / M=2.5 \% \rightarrow M=5 \%$ ) are shown in Fig. 10c. Although the prediction of the simulation results applying a large amount of liquid are worse for all models, the overall results for the models Nos. 2 and 3 are better than in the two previous investigations. These models can predict a fast decline in the fraction retained caused by an intense screen motion very well. In contrast, the results of model No. 1 reveal twice as large deviations as the other two models. In summary, the prediction of simulations with 
operating parameters that have to be extrapolated is far less accurate if model No. 1 is applied instead of the models Nos. 2 and 3.

In the last investigation, five simulation results are predicted with one set of parameters, which have been adjusted to best fit four different configurations. In Fig. 10d, the average deviations of each five predictions to the simulation results are presented. Therein, the green parts of the bars indicate that the values of the operating parameters and the liquid amount of the predicted configurations are located between those of the adjusted ones or have the same values. All models are able to predict these results quite well. The parts of the bars in dark blue and blue show the results for predicting different amplitudes as well as larger and lower liquid amounts, respectively. Results with different frequencies in combination with larger and lower liquid amounts are also predicted and depicted as dark red and red parts of the bars in Fig. 10d, respectively. The models Nos. 2 and 3 predict all these configurations very well, but again model No. 1 reveals larger deviations due to the missing ability to compensate for the opposing subprocesses stratification and passage when values have to be extrapolated.

\section{Conclusions}

In this work, batch screening investigations under the influence of various amounts of liquid have been performed by using the discrete element method (DEM) extended by capillary and viscous forces arising out of the existence of liquid bridge contacts as well as by models for the formation and rupture of liquid bridges. Therein, three particle size classes (coarse, near mesh, fine) as well as various operating parameters (amplitude and frequency) were applied and the resulting values of the fraction retained on the screen surface per size class were compared for the different configurations. The results of the DEM batch screening simulations reveal that larger vibration amplitudes and frequencies predominantly support the decline of the fraction retained for undersized particle classes at least up to the values applied in the investigation here. In contrast, in literature concerning screening with a thin particle bed [45] a lower passage rate is observed for a more intense screen motion due to fewer contacts between the particles and the screen surface leading to a lower number of trials for the particles to pass the apertures. An increase of the liquid amount up to $M=5 \%$ mostly leads to a reduced passage rate and thus to a slower decline of the fraction retained.

From these results, the necessity to study the two subprocesses stratification and passage separately was recognized. Therefore, in a first investigation, the residence time was recorded which the particles need to stratify through the particle bed in the batch screening process. In a further study, the screen surface was replaced by a surface without apertures to analyze the stratification process. The outcomes of both investigations reveal an improvement of the 
stratification process by enlarging the vibration amplitude and frequency and by reducing the liquid amount. Furthermore, the bulk masses were reduced on the initial screen surface to

731

732

733

734

735

736

737 focus on the passage process of a thin particle bed. The obtained results indicate nearly a contrary impact of the operating parameters and the liquid amount on the passage as on the stratification. The particle passage is faster for lower amplitudes and frequencies in the investigated range giving more attempts for the particles to pass the apertures. Furthermore, a larger amount of liquid leads to shorter particle throws giving them more attempts to pass, but also to a hindered passage due to the liquid bridges between the particles and the screen wires.

Additionally, as main novelty of this study, phenomenological screening process models have been extended to represent and predict the results of batch screening simulations under the influence of moisture and different operating conditions. First, a benchmark of the extended models to represent all investigated DEM simulation results with one set of adjusted parameters was performed. Overall, all models reveal larger deviations for representing screening results with low amplitudes or frequencies leading to almost stagnating fraction retained curves. On the contrary, the fitting is more accurate for faster declining curves. The results also indicate that the concurrent subprocesses stratification and passage should be considered in the process models. Therefore, the results of the extended models No. 2 by Subasinghe et al. [48] and No. 3 by Soldinger [50], which both take these subprocesses into account fit the simulation results more accurately than the model No. 1 by Dong et al. [45]. In a final investigation, the capability of the extended process models to predict results of batch screening simulations by applying parameters adjusted for different operating parameters or liquid amounts was tested. All investigated models were able to predict the outcome of simulations where only the liquid amount was different or when the varied operating parameter was located between the ones of the adjusted configurations. In contrast, particularly model No. 1 fails to predict the outcome of screening simulations, where the operating parameters of the adjusted configurations are all larger or all lower. However, the models Nos. 2 and 3 are also able to predict these extrapolated results quite well. Therefore, after an appropriate adjustment to the given properties of the screening apparatus and the material, these models (Nos. 2 and 3) can be used to predict results of batch screening simulations of dry material or of particles under the influence of moisture and are suitable to be integrated in the process simulation framework Dyssol [52] in a further step. Note that the outcome of the process models could also be adjusted and compared with experimental results, applying the same procedure. However, in this investigation, previously validated DEM simulations were used within this innovative method to easier obtain a large range of screening results with various operating parameters, to provide the possibility to easily apply this procedure to different 
screening processes and to give additional information about the subprocesses stratification and passage.

In future investigations, the process models will also be applied for wet screening. For this purpose, some aspects have to be considered. For a small amount of liquid, the screening efficiency decreases with an increase of the liquid amount up to a critical value when the particles are entirely covered by the liquid and the drag forces equal out the adhering forces. From this point on, an increase of the liquid amount results in an increase of the screening efficiency [11]. Therefore, the introduced models have to be applied with varying parameters for the two different regions also realizing for their transition.

\section{Acknowledgements}

The authors gratefully acknowledge the support by DFG within project SPP 1679 through grant number KR3446/7-2 and KR3446/7-3. The original form of the DEM-code "DEM-Calc" applied is based on a development of LEAT, Ruhr-Universität Bochum, Germany. The code "DEMCalc" has then been continuously extended both at Ruhr-Universität Bochum and Technische Universität Berlin, Germany. We thank all who have contributed.

\section{Compliance with ethical standards}

The authors declare that there is no conflict of interest related to this manuscript.

\section{References}

[1] K. Liu, Some factors affecting sieving performance and efficiency, Powder Technol. 193 (2009) 208-213. doi:10.1016/j.powtec.2009.03.027.

[2] V. Grozubinsky, E. Sultanovitch, I.J. Lin, Efficiency of solid particle screening as a function of screen slot size, particle size, and duration of screening - The theoretical approach, Int. J. Miner. Process. 52 (1998) 261-272.

[3] F.S. Guerreiro, R. Gedraite, C.H. Ataíde, Residual moisture content and separation efficiency optimization in pilot-scale vibrating screen, Powder Technol. 287 (2016) 301307. doi:10.1016/j.powtec.2015.10.016.

[4] A. Govender, J.C. van Dyk, Effect of wet screening on particle size distribution and coal properties, Fuel. 82 (2003) 2231-2237. doi:10.1016/S0016-2361(03)00193-5.

[5] J. Robertson, C.J. Thomas, B. Caddy, A.J.M. Lewis, Particle Size Analysis of Soils - A Comparison of Dry and Wet Sieving Techniques, Forensic Sci. Int. 24 (1984) 209-217.

[6] P.A. Cundall, O.D.L. Strack, A discrete numerical model for granular assemblies, Geotechnique. 29 (1979) 47-65.

[7] P.W. Cleary, M.D. Sinnott, R.D. Morrison, Separation performance of double deck banana screens - Part 1: Flow and separation for different accelerations, Miner. Eng. 22 (2009) 1218-1229. doi:10.1016/j.mineng.2009.07.002.

[8] H. Kruggel-Emden, F. Elskamp, Modeling of Screening Processes with the Discrete 
Element Method Involving Non-Spherical Particles, Chem. Eng. Technol. 37 (2014) 847-856. doi:10.1002/ceat.201300649.

[9] P.W. Cleary, P. Wilson, M.D. Sinnott, Effect of particle cohesion on flow and separation in industrial vibrating screens, Miner. Eng. 119 (2018) 191-204. doi:10.1016/j.mineng.2018.01.037.

[10] K.J. Dong, A.B. Yu, Numerical simulation of the particle flow and sieving behaviour on sieve bend/low head screen combination, Miner. Eng. 31 (2012) 2-9. doi:10.1016/j.mineng.2011.10.020.

[11] J.W. Fernandez, P.W. Cleary, M.D. Sinnott, R.D. Morrison, Using SPH one-way coupled to DEM to model wet industrial banana screens, Miner. Eng. 24 (2011) 741-753. doi:10.1016/j.mineng.2011.01.004.

[12] W. Batel, Untersuchungen zur Absiebung feuchter, feinkörniger Haufwerke auf Schwingsieben, Ph.D. thesis, Aachen, 1954.

[13] H.P. Zhu, Z.Y. Zhou, R.Y. Yang, A.B. Yu, Discrete particle simulation of particulate systems: Theoretical developments, Chem. Eng. Sci. 62 (2007) 3378-3396. doi:10.1016/j.ces.2006.12.089.

[14] W. Marquardt, Dynamic Process Simulation - Recent Progress and Future Challenges, in: Chem. Process Control CPC-IV, CACHE Publ., 1991: pp. 131-180.

[15] Y.I. Rabinovich, M.S. Esayanur, B.M. Moudgil, Capillary Forces between Two Spheres with a Fixed Volume Liquid Bridge: Theory and Experiment, Langmuir. 21 (2005) 10992-10997. doi:10.1021/la0517639.

[16] P. Lambert, A. Chau, A. Delchambre, Comparison between Two Capillary Forces Models, Langmuir. 24 (2008) 3157-3163. doi:10.1021/la7036444.

[17] C.D. Willett, M.J. Adams, S.A. Johnson, J.P.K. Seville, Capillary Bridges between Two Spherical Bodies, Langmuir. 16 (2000) 9396-9405.

[18] T. Weigert, S. Ripperger, Calculation of the Liquid Bridge Volume and Bulk Saturation from the Half-filling Angle, Part. Part. Syst. Charact. 16 (1999) 238-242.

[19] J.N. Israelachvili, Intermolecular and Surface Forces, 3rd ed., Academic Press, London, 2011.

[20] J. Fu, M.J. Adams, G.K. Reynolds, A.D. Salman, M.J. Hounslow, Impact deformation and rebound of wet granules, Powder Technol. 140 (2004) 248-257. doi:10.1016/j.powtec.2004.01.012.

[21] B. Crüger, V. Salikov, S. Heinrich, S. Antonyuk, V.S. Sutkar, N.G. Deen, J.A.M. Kuipers, Coefficient of restitution for particles impacting on wet surfaces: An improved experimental approach, Particuology. 25 (2016) 1-9. doi:10.1016/j.partic.2015.04.002.

[22] D. Dopfer, S. Palzer, S. Heinrich, L. Fries, S. Antonyuk, C. Haider, A.D. Salman, Adhesion mechanisms between water soluble particles, Powder Technol. 238 (2013) 35-49. doi:10.1016/j.powtec.2012.06.029.

[23] S. Radl, E. Kalvoda, B.J. Glasser, J.G. Khinast, Mixing characteristics of wet granular matter in a bladed mixer, Powder Technol. 200 (2010) 171-189. doi:10.1016/j.powtec.2010.02.022.

[24] Y. Tsunazawa, D. Fujihashi, S. Fukui, M. Sakai, C. Tokoro, Contact force model including the liquid-bridge force for wet-particle simulation using the discrete element method, Adv. Powder Technol. 27 (2016) 652-660. doi:10.1016/j.apt.2016.02.021. 
[25] P.Y. Liu, R.Y. Yang, A.B. Yu, DEM study of the transverse mixing of wet particles in rotating drums, Chem. Eng. Sci. 86 (2012) 99-107. doi:10.1016/j.ces.2012.06.015.

[26] K. Washino, K. Miyazaki, T. Tsuji, T. Tanaka, A new contact liquid dispersion model for discrete particle simulation, Chem. Eng. Res. Des. 110 (2016) 123-130. doi:10.1016/j.cherd.2016.02.022.

[27] F. Elskamp, H. Kruggel-Emden, DEM simulations of screening processes under the influence of moisture, Chem. Eng. Res. Des. 136 (2018) 593-609. doi:10.1016/j.cherd.2018.06.022.

[28] F. Gabrieli, P. Lambert, S. Cola, F. Calvetti, Micromechanical modelling of erosion due to evaporation in a partially wet granular slope, Int. J. Numer. Anal. Methods Geomech. 36 (2012) 918-943. doi:10.1002/nag.

[29] G. Lian, C. Thornton, M.J. Adams, A Theoretical Study of the Liquid Bridge Forces between Two Rigid Spherical Bodies, J. Colloid Interface Sci. 161 (1993) 138-147.

[30] R.A. Fisher, On the capillary forces in an ideal soil; correction of formulae given by W. B. Haines, J. Agric. Sci. 16 (1926) 492-505.

[31] A. Gladkyy, R. Schwarze, Comparison of different capillary bridge models for application in the discrete element method, Granul. Matter. 16 (2014) 911-920. doi:10.1007/s10035-014-0527-z.

[32] G. Lian, J. Seville, The capillary bridge between two spheres: New closed-form equations in a two century old problem, Adv. Colloid Interface Sci. 227 (2016) 53-62. doi:10.1016/j.cis.2015.11.003.

[33] M.J. Adams, V. Perchard, The Cohesive Forces Between Particles with Interstitial Liquid, Inst. Chem. Eng. Symp. Ser. 91 (1985) 147-160.

[34] O. Pitois, P. Moucheront, X. Chateau, Liquid Bridge between Two Moving Spheres: An Experimental Study of Viscosity Effects, J. Colloid Interface Sci. 231 (2000) 26-31. doi:10.1006/jcis.2000.7096.

[35] A.J. Goldman, R.G. Cox, H. Brenner, Slow viscous motion of a sphere parall to a plane wall-I Motion through a quiescent fluid, Chem. Eng. Sci. 22 (1967) 653-660.

[36] X. Pepin, D. Rossetti, S.M. Iveson, S.J.R. Simons, Modeling the Evolution and Rupture of Pendular Liquid Bridges in the Presence of Large Wetting Hysteresis, J. Colloid Interface Sci. 232 (2000) 289-297. doi:10.1006/jcis.2000.7182.

[37] D. Shi, J.J. McCarthy, Numerical simulation of liquid transfer between particles, Powder Technol. 184 (2008) 64-75. doi:10.1016/j.powtec.2007.08.011.

[38] S. Schmelzle, H. Nirschl, DEM simulations: mixing of dry and wet granular material with different contact angles, Granul. Matter. 20:19 (2018). doi:10.1007/s10035-018-0792-3.

[39] O. Pitois, P. Moucheront, X. Chateau, Rupture energy of a pendular liquid bridge, Eur. Phys. J. B. 23 (2001) 79-86.

[40] S.C. Yang, S.S. Hsiau, The simulation of powders with liquid bridges in a 2D vibrated bed, Chem. Eng. Sci. 56 (2001) 6837-6849. doi:10.1016/S0009-2509(01)00321-9.

[41] R.Y. Yang, R.P. Zou, A.B. Yu, Numerical study of the packing of wet coarse uniform spheres, AIChE J. 49 (2003) 1656-1666. doi:10.1002/aic.690490706.

[42] E.W.C. Lim, Density segregation of dry and wet granular mixtures in vibrated beds, Adv. Powder Technol. 27 (2016) 2478-2488. doi:10.1002/aic.14959.

[43] A. Shimosaka, S. Higashihara, J. Hidaka, Estimation of the sieving rate of powders 
using computer simulation, Adv. Powder Technol. 11 (2000) 487-502. doi:10.1163/156855200750172088.

[44] F. Elskamp, H. Kruggel-Emden, Review and benchmarking of process models for batch screening based on discrete element simulations, Adv. Powder Technol. 26 (2015) 679_ 697. doi:10.1016/j.apt.2014.11.001.

[45] K.J. Dong, B. Wang, A.B. Yu, Modeling of Particle Flow and Sieving Behavior on a Vibrating Screen: From Discrete Particle Simulation to Process Performance Prediction, Ind. Eng. Chem. Res. 52 (2013) 11333-11343. doi:10.1021/ie3034637.

[46] G.K.N.S. Subasinghe, W. Schaap, E.G. Kelly, Modelling the Screening Process: A Probabilistic Approach, Powder Technol. 59 (1989) 37-44.

[47] K. Dong, A.H. Esfandiary, A.B. Yu, Discrete particle simulation of particle flow and separation on a vibrating screen: Effect of aperture shape, Powder Technol. 314 (2017) 195-202. doi:10.1016/j.powtec.2016.11.004.

[48] G.K.N.S. Subasinghe, W. Schaap, E.G. Kelly, Modelling Screening as a Conjugate Rate Process, Int. J. Miner. Process. 28 (1990) 289-300.

[49] M. Soldinger, Interrelation of stratification and passage in the screening process, Miner. Eng. 12 (1999) 497-516.

[50] M. Soldinger, Influence of particle size and bed thickness on the screening process, Miner. Eng. 13 (2000) 297-312.

[51] F. Elskamp, H. Kruggel-Emden, M. Hennig, U. Teipel, Discrete element investigation of process models for batch screening under altered operational conditions, Powder Technol. 301 (2016) 78-95. doi:10.1016/j.powtec.2016.05.039.

[52] V. Skorych, M. Dosta, E.-U. Hartge, S. Heinrich, Novel system for dynamic flowsheet simulation of solids processes, Powder Technol. 314 (2017) 665-679. doi:10.1016/j.powtec.2017.01.061.

[53] H.P. Zhu, Z.Y. Zhou, R.Y. Yang, A.B. Yu, Discrete particle simulation of particulate systems: A review of major applications and findings, Chem. Eng. Sci. 63 (2008) 57285770. doi:10.1016/j.ces.2008.08.006.

[54] A. Munjiza, J.P. Latham, N.W.M. John, 3D dynamics of discrete element systems comprising irregular discrete elements - integration solution for finite rotations in 3D, Int. J. Numer. Methods Eng. 56 (2003) 35-55. doi:10.1002/nme.552.

[55] H. Kruggel-Emden, E. Simsek, S. Rickelt, S. Wirtz, V. Scherer, Review and extension of normal force models for the Discrete Element Method, Powder Technol. 171 (2007) 157-173. doi:10.1016/j.powtec.2006.10.004.

[56] H. Kruggel-Emden, S. Wirtz, V. Scherer, A study on tangential force laws applicable to the discrete element method (DEM) for materials with viscoelastic or plastic behavior, Chem. Eng. Sci. 63 (2008) 1523-1541. doi:10.1016/j.ces.2007.11.025.

[57] H.-J. Butt, M. Kappl, Surface and Interfacial Forces, Wiley-VCH Verlag, Weinheim, 2010.

[58] S.T. Nase, W.L. Vargas, A.A. Abatan, J.J. McCarthy, Discrete characterization tools for cohesive granular material, Powder Technol. 116 (2001) 214-223.

[59] A. Dehghani, A.J. Monhemius, R.J. Gochin, Evaluating the Nakajima et al. model for rectangular-aperture screens, Miner. Eng. 15 (2002) 1089-1094.

[60] N. Standish, The Kinetics of Batch Sieving, Powder Technol. 41 (1985) 57-67. 
933

934

935

936

937

938

939

[61] M. Trumic, N. Magdalinovic, New model of screening kinetics, Miner. Eng. 24 (2011) 42-49. doi:10.1016/j.mineng.2010.09.013.

[62] A.M. Gaudin, Principles of Mineral Dressing, McGraw-Hill, New York, USA, 1939.

[63] F. Elskamp, M. Hennig, H. Kruggel-Emden, U. Teipel, A strategy to determine DEM parameters for spherical and non-spherical particles, Granul. Matter. 19:46 (2017). doi:10.1007/s10035-017-0710-0. 